Comparative Population Studies

Selected Articles in German Vol. 41 (2016): 67-100

(Erstveröffentlichung: 22.08.2017)

\title{
Demografisches Bild der Fertilität in Deutschland vor und nach dem Zensus 2011: Noch keine Trendwende in Sicht
}

\section{Olga Pötzsch}

Zusammenfassung: Die Bevölkerungsvorausberechnungen des Statistischen Bundesamtes zeigen einen voraussichtlichen Rückgang der Geborenenzahl in den 2020er Jahren. Zu dieser Entwicklung wird eine tendenziell rückläufige Anzahl der potenziellen Mütter in Kombination mit einer angenommenen weiterhin niedrigen Geburtenrate führen. Angesichts der vor dem Zensus 2011 vorliegenden empirischen Befunde gab es keine Hinweise darauf, dass eine im nächsten Jahrzehnt eventuell deutlich steigende Geburtenhäufigkeit die sinkende Anzahl potenzieller Mütter kompensieren würde. Der Zensus 2011 hat aber zu Korrekturen in der Bevölkerungsgröße, in der Altersstruktur und folglich in den relativen Maßzahlen der Fertilität wie z.B. der zusammengefassten Geburtenziffer und der endgültigen Kinderzahl je Frau geführt.

Ziel dieses Beitrags ist es, die Auswirkungen des Zensus 2011 auf die Maßzahlen der Fertilität zu quantifizieren und bisherige Befunde zu den Fertilitätstrends auf der zensusjustierten Datengrundlage auf ihre Gültigkeit zu prüfen. Ein besonderes Augenmerk liegt auf den Analysen zur Kohortenfertilität und den Folgen der immer späteren Familiengründung für die endgültige Kinderzahl und die Paritätsverteilung. Anhand von zahlreichen Befunden wird gezeigt, dass ein kontinuierlicher Wiederanstieg der endgültigen Kinderzahl je Frau in den kommenden zwei Jahrzehnten ohne Trendwende im Geburtenverhalten nicht realisierbar ist. Allein um den zunehmenden Rückgang der Fertilität im Alter unter 30 Jahren ab Jahrgang 1974 zu kompensieren und dadurch eine Stabilisierung der endgültigen Kinderzahl auf dem relativ niedrigen Niveau zwischen 1,5 und 1,6 Kindern je Frau zu erreichen, wäre eine verstärkte Zunahme der Fertilität im Alter ab 30 Jahren erforderlich. Ein Anstieg und eine anschließende Stabilisierung der endgültigen Kinderzahl auf dem Niveau von mindestens 1,6 Kindern je Frau würde darüber hinaus eine Trendumkehr in der Entwicklung der Kinderlosenquote und deutliche Veränderungen im Geburtentiming voraussetzen.

Schlagwörter: Aufschub und Nachholen von Geburten · Geburtenentwicklung • Geburtenrate $\cdot$ Kohortenfertilität $\cdot$ Kinderlosigkeit $\cdot$ Zensus 


\section{$1 \quad$ Einleitung}

Nach dem Zensus 2011, der erstmalig nach fast einem viertel Jahrhundert eine Justierung der laufenden Fortschreibung des Bevölkerungsbestands ermöglichte, gehören auch demografische Maßzahlen auf den Prüfstand. Dies betrifft unter anderem die relativen Fertilitätskennzahlen, die neben der absoluten Geborenenzahl die Geburtenentwicklung beschreiben. Sie zeigen die Relation zwischen der Anzahl der geborenen Kinder und der Anzahl der potenziellen Mütter, d.h. der Frauen im gebärfähigen Alter (hier: zwischen 15 und 49 Jahren). Korrigierte Bevölkerungszahlen wirken sich somit über die weibliche Bevölkerung auf die Höhe der Geburtenraten aus (Makrodatenebene). Andererseits stellen sie die Grundgesamtheit für die Hochrechnung des Mikrozensus dar und beeinflussen dadurch seine gewichteten Stichprobenergebnisse (Mikrodatenebene). Diese größte Stichprobenbefragung der privaten Haushalte in Deutschland liefert seit 2008 alle vier Jahre Angaben der Frauen zur Anzahl der geborenen Kinder. Darauf beruhen Befunde zur Struktur der Frauenjahrgänge nach Parität und insbesondere zur Entwicklung der Kinderlosigkeit.

Der vorliegende Beitrag widmet sich der Überprüfung der Geburtentrends unter Berücksichtigung der Auswirkungen des Zensus 2011 auf die wichtigsten Maßzahlen der Fertilität. Der Fokus liegt hier auf den maßgeblichen Aspekten des Geburtenverhaltens, welche aus heutiger Sicht auch für die künftige Fertilitätsentwicklung bedeutsam sein können. Die diese Aspekte charakterisierenden Befunde werden hier analysiert und zu einem Bild der Fertilität zusammengefügt.

Nach der Vorstellung der zugrunde liegenden Datenquellen und Methoden im zweiten Abschnitt des Beitrags werden im dritten Abschnitt Auswirkungen des Zensus 2011 auf die Fertilitätsmessung beschrieben. Die Auswahl der Indikatoren, auf die dabei eingegangen wird, orientiert sich auf die international üblichen und vielfältig verwendeten Kennziffern der Fertilität. Dazu gehören die periodenbezogenen Maßzahlen, wie die Frauenzahl in den Kohorten, die potenzielle Mütter stellen und damit zur Risikobevölkerung gehören, sowie die altersspezifischen und zusammengefassten Geburtenziffern. In der Kohortenperspektive handelt es sich vor allem um die endgültige oder bis zum jeweiligen Alter erreichte durchschnittliche Kinderzahl sowie um die Paritätsverteilung in den Frauenjahrgängen. Die Momentaufnahme des Geburtenniveaus im Jahr 2011 vor und nach dem Zensus ist der Ausgangspunkt für die Justierung der Fertilitätstrends und der Annahmen zu Bevölkerungsprojektionen.

Daran knüpft der vierte Abschnitt an, in dem gegenwärtige Geburtentrends und Überlegungen zur künftigen Fertilitätsentwicklung auf der neuen Datenbasis nach dem Zensus erörtert werden. Während sich die Zahl der potenziellen Mütter in den kommenden zwei Jahrzehnten weitgehend aus der bisherigen demografischen Entwicklung ergibt, muss die künftige Geburtenhäufigkeit auf Grundlage von Befunden zum Geburtenverhalten geschätzt werden. Ein hierfür gewählter Ansatz beruht auf Untersuchungen der Kohortenfertilität. Zentrale Frage ist dabei die Entwicklung der endgültigen Kinderzahl je Frau unter dem Einfluss der immer späteren Familiengründung. Die folgenden Analysen knüpfen an die Untersuchungen zum Aufschub und Nachholen der Geburten an (Frejka/Calot 2001; Frejka/Sobotka 2008; Sobot- 
ka et al. 2011; Frejka 2012; Pötzsch 2013), zur Interaktion zwischen den Paritäten und ihrer Auswirkung auf die endgültige Kinderzahl (Statistisches Bundesamt 2009, 2013; Sobotka 2011; Bujard/Lück 2015a) sowie zur Entwicklung der Kinderlosigkeit (Dorbritz/Ruckdeschel 2007; Sobotka 2011; Konizka/Kreyenfeld 2013, Statistisches Bundesamt 2013, 2015a; Beier et al. 2012; te Velde et al. 2012).

Die bisher veröffentlichten Vorausschätzungen der endgültigen Kinderzahl reichen bis zu den Mitte beziehungsweise Ende der 1970er geborenen Jahrgängen (Goldstein/Kreyenfeld 2011; Pötzsch 2010a, 2013; Myrskylä et al. 2013; Statistisches Bundesamt 2015a). Dabei wird nicht in Frage gestellt, dass der rückläufige Trend bei den Ende der 1960er Jahre geborenen Frauen erst einmal endet und durch einen geringfügigen Anstieg der endgültigen Kinderzahl bis zur Kohorte 1973 abgelöst wird. Danach scheint die Kohortenfertilität in Westdeutschland zu stagnieren, während sie in Ostdeutschland noch weiter steigen dürfte (vgl. Pötzsch 2013: 95-97; Myrskylä et al. 2013, Table 2 und Appendix Figure 1). Im Folgenden werden die gesamtdeutschen Fertilitätstrends auf Grundlage der zensusjustierten Ergebnisse überprüft und Perspektiven der Fertilitätsentwicklung bis zum Jahrgang 1984 erörtert.

\section{Daten und Methoden}

Die relativen Maßzahlen der Fertilität beziehen sich in der Regel auf die jahresdurchschnittliche Bevölkerung (weiter auch durchschnittliche Bevölkerung genannt). Im Unterschied zur Stichtagsbevölkerung berücksichtigt diese unterjährige Schwankungen der Bevölkerungszahl, die in der Regel vor allem aufgrund von Wanderungen entstehen. Die durchschnittliche Bevölkerung bietet damit eine bessere Annäherung an die sogenannte Risikobevölkerung als Bezugsgröße für Fertilitätsmaßzahlen. Die Risikobevölkerung ist dabei eine Schätzung der „Personenjahre“ oder der Lebenszeit einer Population, in der diese Population „dem Risiko einer Geburt” ausgesetzt war (Luy 2016: 123-125). Für die Berechnung der altersspezifischen Geburtenziffern wird zudem die durchschnittliche Bevölkerung nach einzelnen Altersjahren benötigt. Die amtliche Statistik verwendet hierfür zwei Berechnungsmethoden: nach Alters- und nach Geburtsjahren. In diesem Beitrag wird die Bevölkerung nach Geburtsjahren (oder Kohorten) verwendet, da dies eine Langzeitbetrachtung ermöglicht. Die Ergebnisse der Geburtenstatistik nach dem vollendeten Alter liegen dagegen erst seit 2000 vor.

Um das Verständnis der weiteren Ausführungen zu erleichtern, sei kurz daran erinnert, dass unter Kohortenfertilität in der Regel die endgültige Zahl der Kinder verstanden wird, die ein Frauenjahrgang im Durchschnitt zur Welt gebracht hat. Die endgültige durchschnittliche Kinderzahl der Frauenkohorten (cohort total fertility rate, CTFR) wird berechnet als Summe der altersspezifischen Geburtenziffern eines Frauenjahrgangs, die in den Kalenderjahren nachgewiesen wurden, in denen der entsprechende Jahrgang die Altersstufen von 15 bis zu 49 Jahren durchlief. Die altersspezifische Geburtenziffer zeigt dabei die in einem Kalenderjahr nachgewiesene Relation zwischen der Zahl der Geborenen von Müttern einer Kohorte $c$ im jeweils 
erreichten Alter, $B_{C}(x)$, und der Zahl aller Frauen dieser Kohorten im gleichen Alter, $P_{c}(x)$ :

$$
\text { CTFR }=\sum_{\alpha}^{\beta} f_{c}(x)=\sum_{\alpha}^{\beta} \frac{B_{c}(x)}{P_{c}(x)}
$$

Der additive Charakter der CTFR erlaubt es, diese nach Altersspannen zu unterteilen, die Parameter $\alpha$ und $\beta$ werden dabei variiert. In diesem Beitrag wird diese Eigenschaft genutzt, um die unterschiedlichen altersspezifischen Trends im jeweils jüngeren und höheren fertilen Alter sowie die Interaktion zwischen dem Aufschub und dem Nachholen der Geburten abzubilden. Dabei werden z.B. einerseits die altersspezifischen Geburtenziffern der Frauenkohorten im Alter von 15 bis 29 Jahren und andererseits im Alter von 30 bis 49 Jahren addiert. Die Summe der beiden Ergebnisse ergibt die durchschnittliche endgültige Kinderzahl:

$$
\sum_{15}^{29} f_{c}(x)+\sum_{30}^{49} f_{c}(x)=\sum_{15}^{49} f_{c}(x)=C T F R
$$

Die Analyse der Kohortenfertilität in Abschnitt 4.1 ist an einen Ansatz von Frejka und Calot (2001) und seine Weiterentwicklung von Sobotka et al. (2011) angelehnt. Dieser Ansatz zur Berechnung des Index der nachgeholten Geburten (Recuperation Index) wurde hier vereinfacht und leicht modifiziert mit dem Ziel, die Entwicklung vom Aufschub und Nachholen der Fertilität möglichst anschaulich darzustellen. Die beiden Prozesse werden dabei separat betrachtet und miteinander verglichen. Im Vorfeld werden die Abweichungen zwischen den kumulierten altersspezifischen Geburtenziffern der betrachteten Kohorte und der als Referenz gewählten Kohorte für jedes Altersjahr gemessen. Die größte Abweichung markiert den "Tiefpunkt", in dem sich die kumulierte Fertilität der betrachteten Kohorte am stärksten von der Referenzkohorte unterscheidet. Nachdem der Tiefpunkt überschritten ist, beginnt die Nachholphase. Werden die aufgeschobenen Geburten nicht vollständig nachgeholt, bleibt auch am Ende der reproduktiven Phase eine "Restabweichung" zwischen den endgültigen Kinderzahlen. Wenn die Abweichung im Tiefpunkt durch das Nachholen im höheren Alter kompensiert oder überkompensiert wird, dann wird die endgültige Kinderzahl der betrachteten Kohorte im Vergleich zur abgeschlossenen Fertilität der Referenzkohorte jeweils gleich oder höher sein. (Mehr zum BasisBezugsmodel und Anwendungsbeispiel für Deutschland s. bei Sobotka et al. 2011, Abschnitt 3.2 sowie Pötzsch 2013).

Die endgültige Kinderzahl der Frauenkohorten kann theoretisch auch auf Basis der Mikrodaten, d.h. der Angaben der Befragten im Mikrozensus zur Zahl der geborenen Kinder, ermittelt werden. Dabei wird die Gesamtzahl der von Frauen eines Jahrgangs geborenen Kinder durch die Zahl der Frauen dieses Jahrgangs dividiert. Diese durchschnittliche Kinderzahl ist jedoch nur bedingt mit der CTFR vergleichbar. Einerseits beziehen sich diese beiden Indikatoren auf unterschiedliche Populationen (Pötzsch 2010b: 171-172; Kreyenfeld et al. 2012: 394). Zum Beispiel war die Zusammensetzung des Frauenjahrgangs 1950 zum Zeitpunkt der Mikrozensusbefragung im Jahr 2012 aufgrund von Wanderungen und Sterbefällen mit 
hoher Wahrscheinlichkeit eine andere als in den Jahren 1965 bis 1999, aus denen die 35 altersspezifischen Geburtenziffern dieses Jahrgangs (zwischen seinem 16. und 50. Lebensjahr) stammen. Andererseits sind die Mikrozensusergebnisse mit einem Stichprobenfehler behaftet und können aufgrund von Antwortausfällen verzerrt sein. Um systematische Verzerrungen aufgrund von Antwortausfällen zu minimieren, wird ein Imputationsverfahren angewendet (Statistisches Bundesamt 2009, 2013). Trotzdem bleibt ein Teil der Fälle unbekannt. Im Mikrozensus 2012 betrifft das vor allem die Verteilung der Mütter nach Zahl der geborenen Kinder. Bei der Berechnung von durchschnittlichen Kinderzahlen müsste darauf besonders geachtet werden. Wenn nur bekannte Fälle einbezogen werden, wird die Anzahl der Mütter ohne Angabe zur Kinderzahl proportional auf alle Paritäten einschließlich der Parität 0, d.h. ohne Kind verteilt. Bei so einem Durchschnittswert ist das Gewicht der Kinderlosen - methodisch bedingt - erhöht. Zudem variiert der prozentuale Anteil der fehlenden Angaben zur Zahl der geborenen Kinder je nach Jahrgang z.B. im Mikrozensus 2012 zwischen $1 \%$ und 8 \%, was die Vergleichbarkeit der Ergebnisse zwischen den Kohorten zusätzlich einschränkt.

Trotz dieser Einschränkungen ist der Mikrozensus die wichtigste Datenquelle für die Bestimmung des Kinderlosenanteils und die Verteilung der Mütter nach Kinderzahl. Auch für die Einschätzung der Auswirkungen der zensusbedingten Korrektur der Bevölkerung auf die Kohortenfertilität wird hier der Mikrozensus herangezogen. Dabei werden die Resultate der Befragung im Jahr 2012 auf Grundlage der Hochrechnung vor und nach dem Zensus miteinander verglichen. Da sich die zu vergleichenden Ergebnisse auf dieselben Jahrgänge beziehen und auf den gleichen Stichprobenangaben beruhen, kann auf dieser Grundlage auch die durchschnittliche Kinderzahl je Kohorte vor und nach dem Zensus verglichen werden. Dabei geht es weniger um die Höhe der durchschnittlichen Kinderzahl je Frau, sondern um das Ausmaß der Verschiebungen infolge der neuen Hochrechnung.

Für viele Indikatoren ist es nach wie vor sinnvoll, die Entwicklung nicht nur für Deutschland insgesamt, sondern auch für West- und Ostdeutschland abzubilden. Trotz Annährungstendenzen sind Unterschiede im Geburtenverhalten der Frauen immer noch deutlich (Statistisches Bundesamt 2013). Dabei wird Berlin, obwohl es geografisch im Osten Deutschlands liegt, nicht zu den ostdeutschen Ländern gerechnet. Ursächlich dafür ist, dass für Berlin ein eher westliches Fertilitätsmuster charakteristisch ist. Das Einbeziehen von Berlin in die Ergebnisse für Ostdeutschland würde aufgrund seines großen Gewichts die ostdeutschen Geburtentrends verwässern und den Vergleich mit den westlichen Bundesländern verzerren. In den Ergebnissen für Deutschland ist Berlin enthalten. 


\section{Auswirkungen des Zensus auf die wichtigsten Fertilitätsmaße}

\subsection{Weibliche Bevölkerung als Bezugsgröße für die Geburtenraten}

Die Gesamtzahl der potenziellen Mütter im Jahr 2011 - d.h. Frauen, die im Alter zwischen 15 und 49 Jahren waren - sank in der zensusjustierten Bevölkerung um 321.000 oder $1,8 \%$ (Tab. 1). In Westdeutschland war die Korrektur mit lediglich $-1,5 \%$ geringer als in den neuen Ländern: $-2,0 \%$. Während die Anzahl der Frauen mit deutscher Staatsangehörigkeit fast unverändert blieb, bestand bei den Frauen mit ausländischer Staatsangehörigkeit ein verhältnismäßig hoher Korrekturbedarf. Die Anzahl der Ausländerinnen im Alter zwischen 15 und 49 Jahren wurde in Deutschland insgesamt um $-11,4 \%$, im früheren Bundesgebiet um $-10,3 \%$ und in den neuen Ländern um -19,0 \% korrigiert. Dies führte zum Sinken des Anteils der Ausländerinnen an allen Frauen im gebärfähigen Alter von 12,4 \% auf $11,1 \%$.

Tab. 1: Frauen zwischen 15 und 49 Jahren - Korrekturbedarf nach dem Zensus 2011

\begin{tabular}{|c|c|c|c|c|}
\hline \multirow[t]{3}{*}{ Gebiet } & \multicolumn{2}{|c|}{ Jahresdurchschnittliche Bevölkerung 2011} & \multicolumn{2}{|c|}{$\begin{array}{l}\text { Veränderung nach } \\
\text { dem Zensus }\end{array}$} \\
\hline & VOR Zensus & NACH Zensus & absolut & relativ \\
\hline & \multicolumn{2}{|c|}{ 1.000 Personen } & & $\%$ \\
\hline \multicolumn{5}{|l|}{ Frauen insgesamt } \\
\hline Deutschland & 18.209 & 17.888 & -321 & $-1,8$ \\
\hline Früheres Bundesgebiet ${ }^{1}$ & 14.810 & 14.589 & -221 & $-1,5$ \\
\hline Neue Länder ${ }^{1}$ & 2.554 & 2.504 & -50 & $-2,0$ \\
\hline \multicolumn{5}{|l|}{ Deutsche Frauen } \\
\hline Deutschland & 15.959 & 15.895 & -64 & $-0,4$ \\
\hline Früheres Bundesgebiet ${ }^{1}$ & 12.811 & 12.797 & -14 & $-0,1$ \\
\hline Neue Länder ${ }^{1}$ & 2.461 & 2.429 & -32 & $-1,3$ \\
\hline \multicolumn{5}{|l|}{ Aus/änderinnen } \\
\hline Deutschland & 2.250 & 1.993 & -257 & $-11,4$ \\
\hline Früheres Bundesgebiet ${ }^{1}$ & 1.999 & 1.792 & -207 & $-10,3$ \\
\hline Neue Länder ${ }^{1}$ & 92 & 75 & -18 & $-19,0$ \\
\hline
\end{tabular}

1 Ohne Berlin-West bzw. ohne Berlin-Ost.

Quelle: Statistisches Bundesamt, Bevölkerungsfortschreibung. Eigene Berechnungen

Für die altersspezifischen Geburtenziffern sind Veränderungen in den einzelnen Geburtskohorten innerhalb der gebärfähigen Phase relevant. Deutschlandweit ergibt sich ein relativ klares Muster (Abb. 1). Überdurchschnittlich ist die Korrektur der Anzahl der Frauen im Alter zwischen 19 und 27 Jahren ausgefallen (bis -2,5\%). Wie aus dem unteren Segment in Abb. 1 ersichtlich, gehen diese Änderungen vor allem auf die Korrekturen bei den Frauen mit ausländischer Staatsangehörigkeit zurück. Bei den Frauen mit deutscher Staatsangehörigkeit sind dagegen die Abweichungen 
Abb. 1: Frauen zwischen 15 und 49 Jahren $^{1}$ - relative Veränderung der jahresdurchschnittlichen Bevölkerung 2011 nach dem Zensus
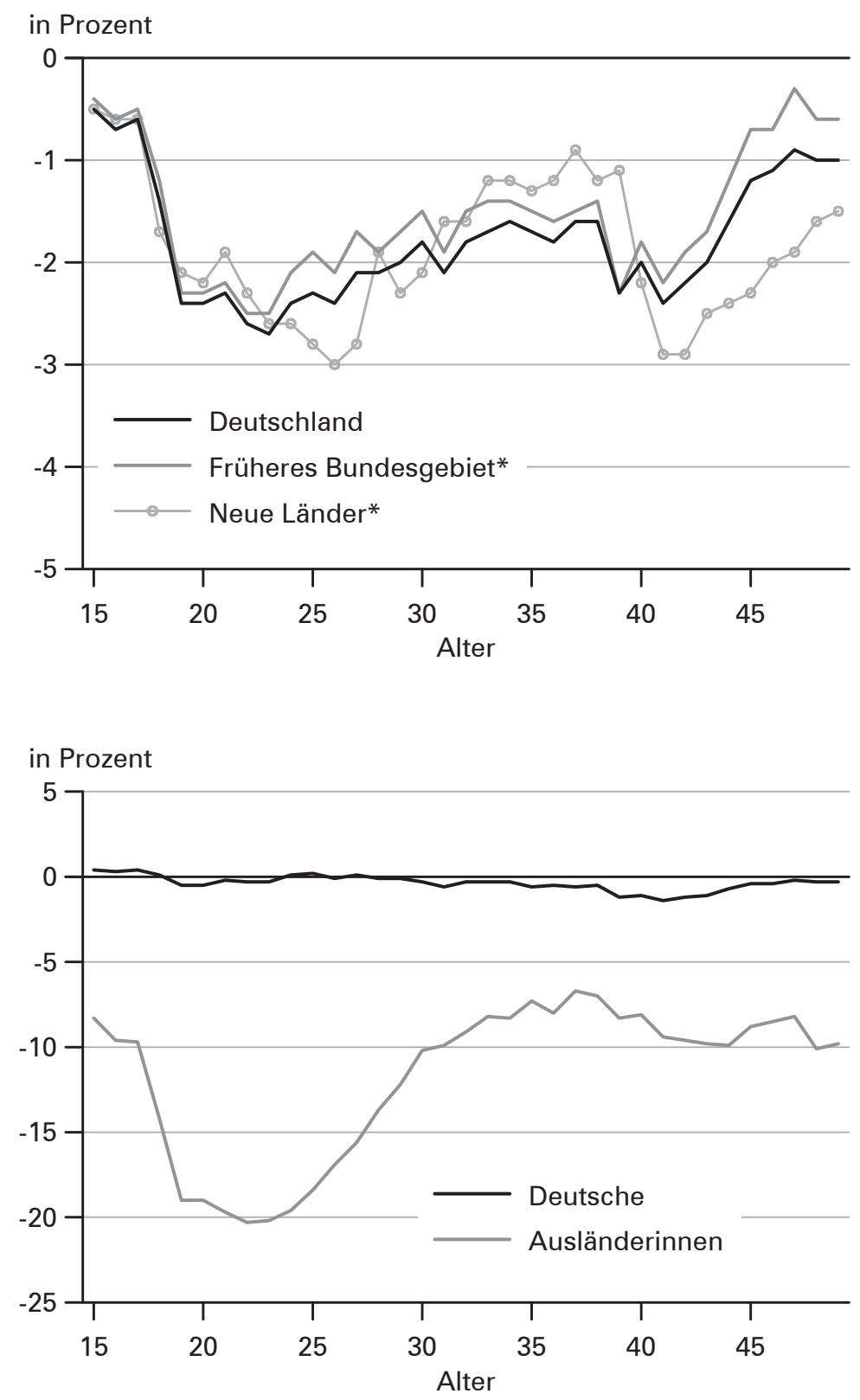

${ }^{1}$ Im Jahr 2011 erreichtes Alter.

* Ohne Berlin-West bzw. ohne Berlin-Ost.

Quelle: Statistisches Bundesamt, Bevölkerungsfortschreibung. Eigene Berechnungen und Darstellung 
vernachlässigbar gering. In den neuen Ländern ergab sich zudem ein etwas gröBerer Korrekturbedarf für die Anzahl der Frauen im Alter von Anfang bis Mitte 40. Da aber die Geburtenhäufigkeit in diesem Alter gering ist, dürfte diese Änderung die zusammengefasste Geburtenziffer 2011 nur leicht beeinflussen. Zugleich ist das möglicherweise ein Indiz dafür, dass in der Bestandsfortschreibung direkt nach der deutschen Vereinigung die Fortzüge der damals 20- bis 25-Jägrigen wegen fehlender Abmeldungen nicht vollständig berücksichtigt wurden und die Frauenzahl in den entsprechenden Kohorten etwas zu hoch ausgewiesen wurde.

Für die Maßzahlen der Kohorten, wie z.B. endgültige Kinderzahl je Frau, ist darüber hinaus von Interesse, wie die Korrekturen für die älteren Frauenjahrgänge ausgefallen sind. Die Bereinigungen bei den Jahrgängen 1930 bis 1961 - für diese Geburtskohorten liegen Geburtenziffern vor - waren mit durchschnittlich -0,9\% deutlich geringer als bei den jüngeren Frauen. Wir können nicht ausschließen, dass die Unsicherheiten in den einzelnen Jahren zwischen den Zensen, als die Frauen dieser Kohorten im gebärfähigen und zugleich mobilen Alter waren, teilweise grö-

Abb. 2: Frauen zwischen 15 und 49 Jahren $^{1}$ - relative Veränderungen in der jahresdurchschnittlichen Bevölkerung 2011 nach dem Zensus nach Bundesländern

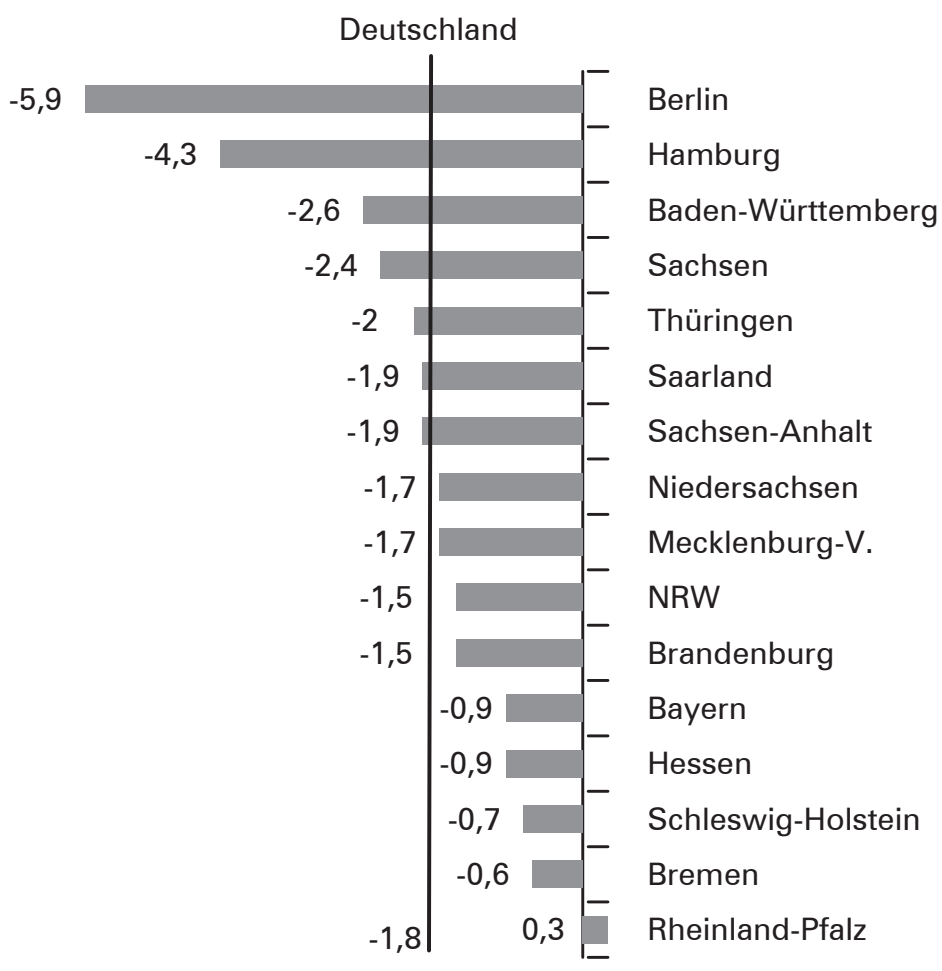

${ }^{1}$ Im Jahr 2011 erreichtes Alter.

Quelle: Statistisches Bundesamt, Bevölkerungsfortschreibung. Eigene Berechnungen und Darstellung 
Ber waren. Trotzdem ist die geringe Abweichung zum Zeitpunkt des Zensus 2011 ein wichtiger Hinweis darauf, dass die kohortenspezifischen Geburtenziffern offenbar keine großen Verzerrungen enthalten.

In den einzelnen Bundesländern war der Korrekturbedarf unterschiedlich groß und variierte zwischen $+0,3 \%$ in Rheinland-Pfalz bis zu $-5,9 \%$ in Berlin (Abb. 2). Die Stadtstaaten Berlin und Hamburg verzeichneten die größten Abweichungen zwischen dem Zensusergebnis und der Bevölkerungsfortschreibung.

\subsection{Auswirkungen des Zensus auf Fertilitätsmaßzahlen des Jahres 2011}

Bei der Berechnung der Geburtenziffern verändert sich durch die Zensusjustierung nur die Bezugsgröße - die weibliche Bevölkerung - im Nenner der Formel (1). Die Zahl der Geborenen im Zähler bleibt dagegen unverändert. Die Zensuskorrektur wirkt sich deshalb auf die relativen Geburtenkennzahlen in einem vergleichbaren Verhältnis zur Korrektur der Frauenzahl aus, nur mit umgekehrtem Vorzeichen. Besonderes Interesse gilt dabei aus demografischer Sicht den altersspezifischen und aggregierten Geburtenziffern, wie z.B. der zusammengefassten Geburtenziffer (aus der Periodensicht) und der endgültigen beziehungsweise bis zum jeweiligen Alter erreichen Kinderzahl je Frau (aus der Kohortenperspektive).

Die zusammengefasste Geburtenziffer betrug in Deutschland 2011 unter Berücksichtigung der Zensuskorrektur 1,39 Kinder je Frau (Tab. 2). Dieser Wert war um 2,0 \% höher als vor dem Zensus: 1,36 Kinder je Frau. Im früheren Bundesgebiet betrug die Veränderung 1,8 \% (von 1,36 auf 1,38 Kinder je Frau) und in den neuen Ländern 2,0 \% (von 1,43 auf 1,46 Kinder je Frau).

Für Frauen mit deutscher Staatsangehörigkeit waren die Auswirkungen der Bevölkerungskorrekturen auf die zusammengefasste Geburtenziffer mit durchschnittlich $0,3 \%$ kaum spürbar. Bei Frauen mit ausländischer Staatsangehörigkeit stieg diese dagegen von 1,58 auf 1,82 Kinder je Frau um $15 \%$.

In den einzelnen Bundesländern betrugen die Korrekturen der Geburtenrate von $0,5 \%$ in Rheinland-Pfalz bis $6,3 \%$ in Berlin. Zu den Bundesländern mit einem besonders großen Anpassungsbedarf gehörte neben Berlin auch Hamburg (4,6 \%). Ursächlich dafür war u.a. der hohe Anteil der Ausländerinnen an der weiblichen Bevölkerung in beiden Stadtstaaten (bei Frauen im Alter zwischen 15 und 49 Jahren jeweils rund $16 \%$ ). Da die ausländische Bevölkerung nach dem Zensus deutlich reduziert wurde, stiegen die Geburtenraten in den Stadtstaaten überdurchschnittlich stark.

Die altersspezifischen Geburtenziffern für Frauen insgesamt haben sich nur geringfügig verändert. Die Verteilung der Geburtenhäufigkeiten nach dem Alter der Mutter blieb deshalb nahezu konstant. Das durchschnittliche Gebäralter in Deutschland betrug auch nach der Zensuskorrektur 30,5 Jahre, der Median lag bei 31 Jahren. Allerdings stieg die höchste Fertilitätsrate, die der 31-Jährigen, von ursprünglich 98 auf 100 Kinder je 1000 Frauen. Im früheren Bundesgebiet und in den neuen Ländern war ebenfalls nur eine leichte Zunahme der maximalen Häufigkeit zu verzeichnen.

Die altersspezifischen Veränderungen bei den Frauen mit ausländischer Staatsangehörigkeit entfielen insbesondere auf das Alter um Mitte zwanzig (Abb. 3). Dies 
Tab. 2: Zusammengefasste Geburtenziffer 2011 - Korrekturbedarf nach dem Zensus 2011

\begin{tabular}{|c|c|c|c|c|}
\hline \multirow[t]{2}{*}{ Gebiet/Staatsangehörigkeit } & \multirow[t]{2}{*}{ VOR Zensus } & \multirow{2}{*}{$\begin{array}{l}\text { NACH Zensus } \\
\text { Kinder je Frau }\end{array}$} & \multicolumn{2}{|c|}{ Veränderung } \\
\hline & & & absolut & $\begin{array}{c}\text { relativ } \\
\%\end{array}$ \\
\hline \multicolumn{5}{|l|}{ Frauen insgesamt } \\
\hline Deutschland & 1,36 & 1,39 & 0,03 & 2,0 \\
\hline Früheres Bundesgebiet ${ }^{1}$ & 1,36 & 1,38 & 0,02 & 1,8 \\
\hline Neue Länder ${ }^{1}$ & 1,43 & 1,46 & 0,03 & 2,0 \\
\hline \multicolumn{5}{|l|}{ Deutsche Frauen } \\
\hline Deutschland & 1,33 & 1,34 & 0,00 & 0,3 \\
\hline Früheres Bundesgebiet ${ }^{1}$ & 1,32 & 1,32 & 0,00 & 0,1 \\
\hline Neue Länder ${ }^{1}$ & 1,43 & 1,45 & 0,02 & 1,2 \\
\hline \multicolumn{5}{|l|}{ Ausländerinnen } \\
\hline Deutschland & 1,58 & 1,82 & 0,24 & 15,0 \\
\hline Früheres Bundesgebiet ${ }^{1}$ & 1,60 & 1,81 & 0,22 & 13,5 \\
\hline Neue Länder ${ }^{1}$ & 1,48 & 1,82 & 0,34 & 22,8 \\
\hline \multicolumn{5}{|l|}{ Nach Bundes/ändern } \\
\hline Baden-Württemberg & 1,36 & 1,39 & 0,04 & 2,8 \\
\hline Bayern & 1,34 & 1,36 & 0,01 & 1,0 \\
\hline Berlin & 1,31 & 1,40 & 0,08 & 6,3 \\
\hline Brandenburg & 1,41 & 1,44 & 0,02 & 1,6 \\
\hline Bremen & 1,27 & 1,29 & 0,01 & 1,1 \\
\hline Hamburg & 1,26 & 1,32 & 0,06 & 4,6 \\
\hline Hessen & 1,39 & 1,40 & 0,01 & 0,6 \\
\hline Mecklenburg-Vorpommern & 1,42 & 1,44 & 0,02 & 1,5 \\
\hline Niedersachsen & 1,39 & 1,42 & 0,04 & 2,6 \\
\hline Nordrhein-Westfalen & 1,36 & 1,39 & 0,02 & 1,8 \\
\hline Rheinland-Pfalz & 1,37 & 1,37 & 0,01 & 0,5 \\
\hline Saarland & 1,28 & 1,31 & 0,04 & 2,8 \\
\hline Sachsen & 1,48 & 1,51 & 0,04 & 2,5 \\
\hline Sachsen-Anhalt & 1,40 & 1,43 & 0,03 & 2,0 \\
\hline Schleswig-Holstein & 1,38 & 1,40 & 0,02 & 1,5 \\
\hline Thüringen & 1,41 & 1,44 & 0,03 & 1,9 \\
\hline
\end{tabular}

1 Ohne Berlin-West bzw. ohne Berlin-Ost.

Quelle: Statistisches Bundesamt, Geburtenstatistik. Eigene Berechnungen 
führte zu einer leichten Absenkung des durchschnittlichen Gebäralters um 0,3 Jahre auf 29,3 Jahre und des Medians um 0,4 Jahre auf 29,6 Jahre. Die maximale Geburtenhäufigkeit (im Alter von 29 Jahren) stieg gleichzeitig von 99 auf 113 Kinder je 1000 Frauen. Für die Fertilität aller Frauen insgesamt haben diese Veränderungen jedoch keine relevanten Auswirkungen. Abbildung 3(a) veranschaulicht deutlich, dass sie lediglich zu einer marginalen Verschiebung im Verlauf der altersspezifischen Geburtenraten führen.

Abb. 3: Altersspezifische Geburtenziffern 2011- Korrekturbedarf nach dem Zensus 2011
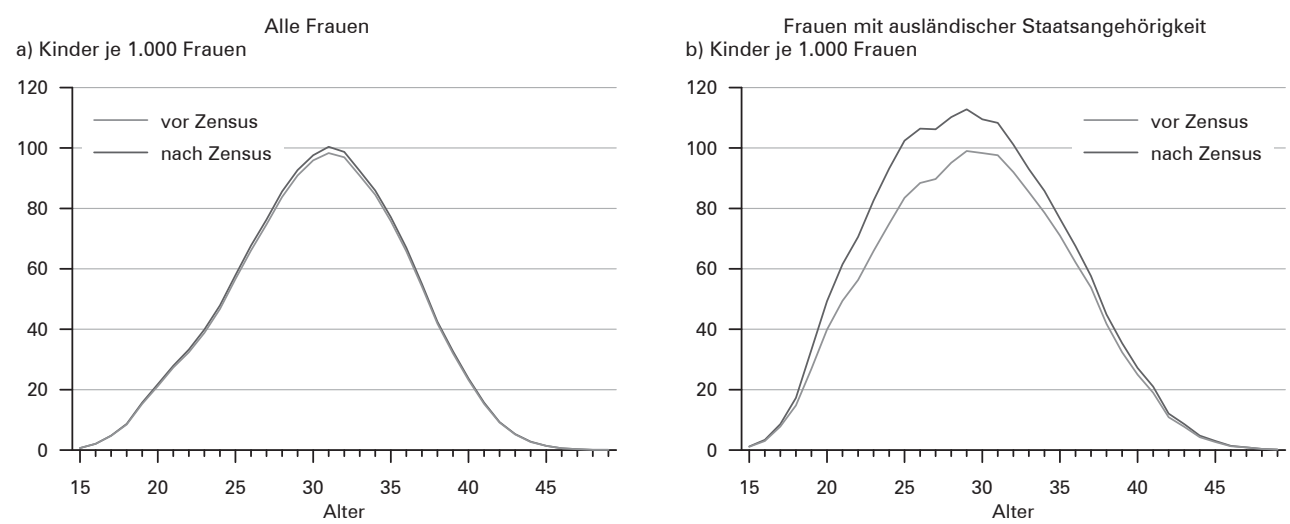

Quelle: Statistisches Bundesamt, Geburtenstatistik. Eigene Berechnungen und Darstellung

\subsection{Auswirkungen des Zensus auf die Zeitreihen der Periodenfertilität}

Für die langfristige Entwicklung der zusammengefassten Geburtenziffer ergibt sich durch die zensusbedingte Justierung keine spürbare Niveauverschiebung. Die in Abbildung 4 dargestellten Kurven enthalten für das Jahr 2011 sowohl die Werte vor als auch nach der Berücksichtigung der Zensusergebnisse. Die neuen korrigierten Werte fügen sich in die Verläufe ohne deutliche Sprünge ein. Auch beim Vergleich der jährlichen Veränderungen in der zusammengefassten Geburtenziffer wird deutlich, dass der "Zensus-Effekt" kaum stärker ausgefallen ist als die üblichen jährlichen Schwankungen.

Bei Frauen mit deutscher Staatsangehörigkeit ist der "Zensus-Effekt" auf die Entwicklung der TFR vernachlässigbar gering, diese bleibt auf dem niedrigen Niveau: 1,34 Kinder je Frau statt 1,33 Kinder je Frau vor dem Zensus (Abb. 5). Bei Frauen mit ausländischer Staatsangehörigkeit führte dagegen die Zensusjustierung zu einer sichtbaren Niveauverschiebung in der TFR des Jahres 2011 von 1,58 auf 1,82 Kinder je Frau. Dadurch ergibt sich die Notwendigkeit einer Trendkorrektur und die These über eine schnelle Annährung der Geburtenrate der Ausländerinnen an die der Deutschen muss relativiert werden. Der Rückgang der zusammengefassten Geburtenziffer der Ausländerinnen zwischen 1991 und 2011 ist nach den neuen Erkenntnissen mit 11 \% nur halb so stark ausgefallen, wie ursprünglich angenommen 
Abb. 4: Zusammengefasste Geburtenziffer 1990 bis 2013 mit „Zensus-Effekt” im Jahr 2011

Kinder je Frau

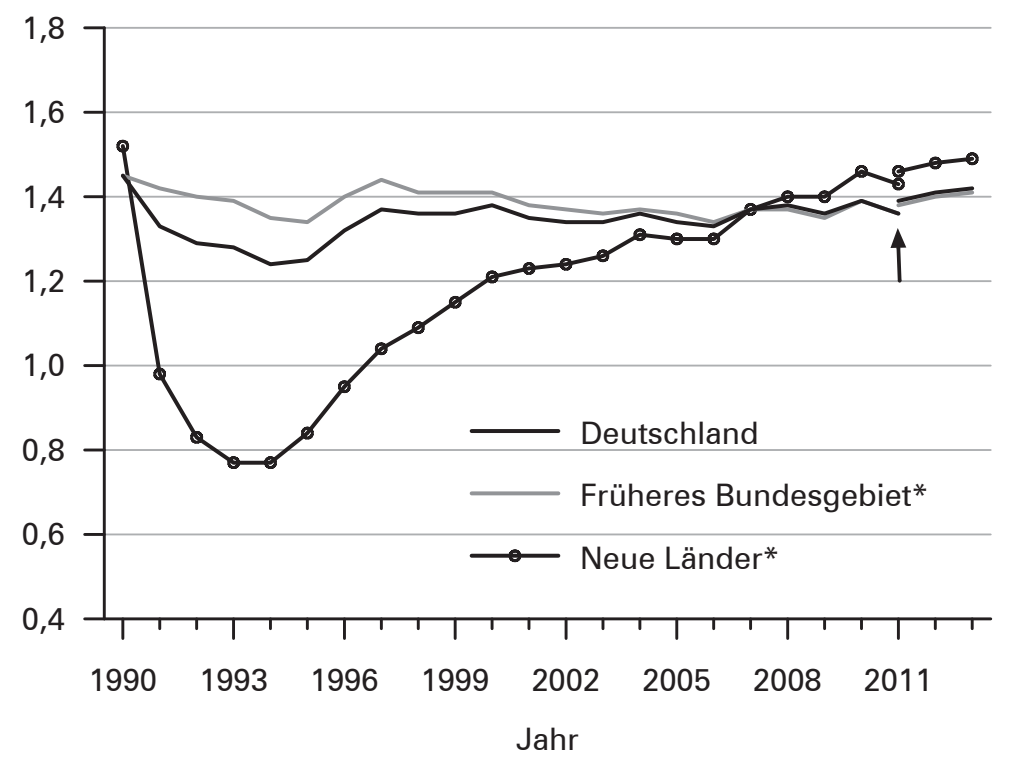

Abb. 5: Zusammengefasste Geburtenziffer nach Staatsangehörigkeit der Frauen 1991 bis 2013 mit „Zensus-Effekt“ im Jahr 2011

Kinder je Frau

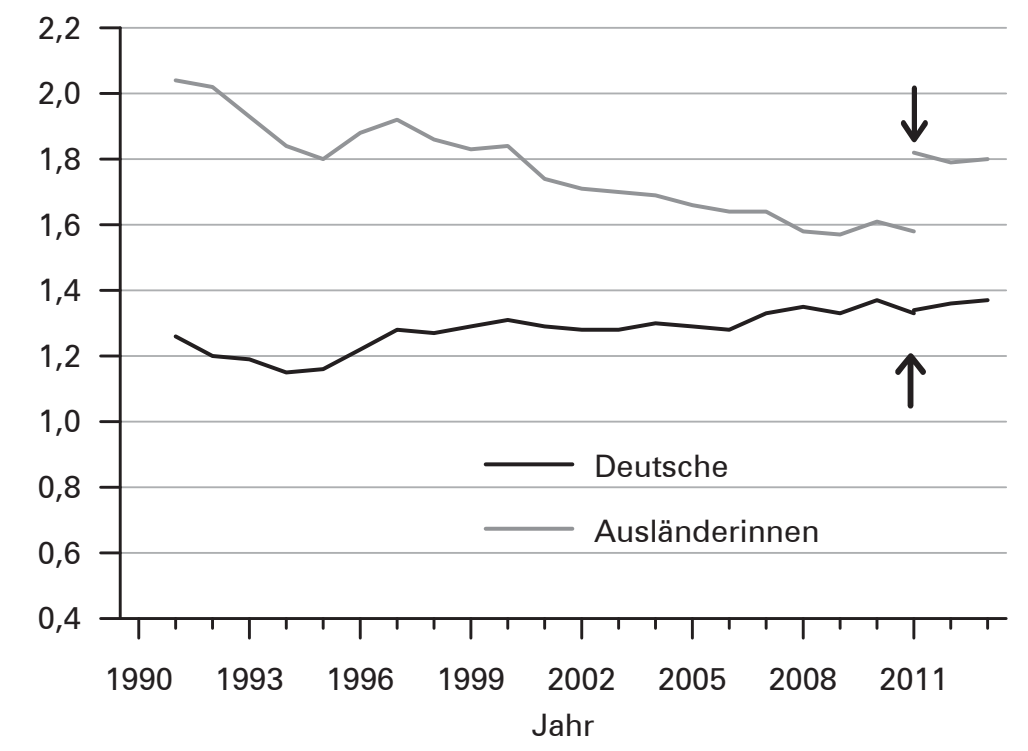

* Ohne Berlin-West bzw. ohne Berlin-Ost.

Quelle: Statistisches Bundesamt, Geburtenstatistik. Eigene Berechnungen und Darstellung 


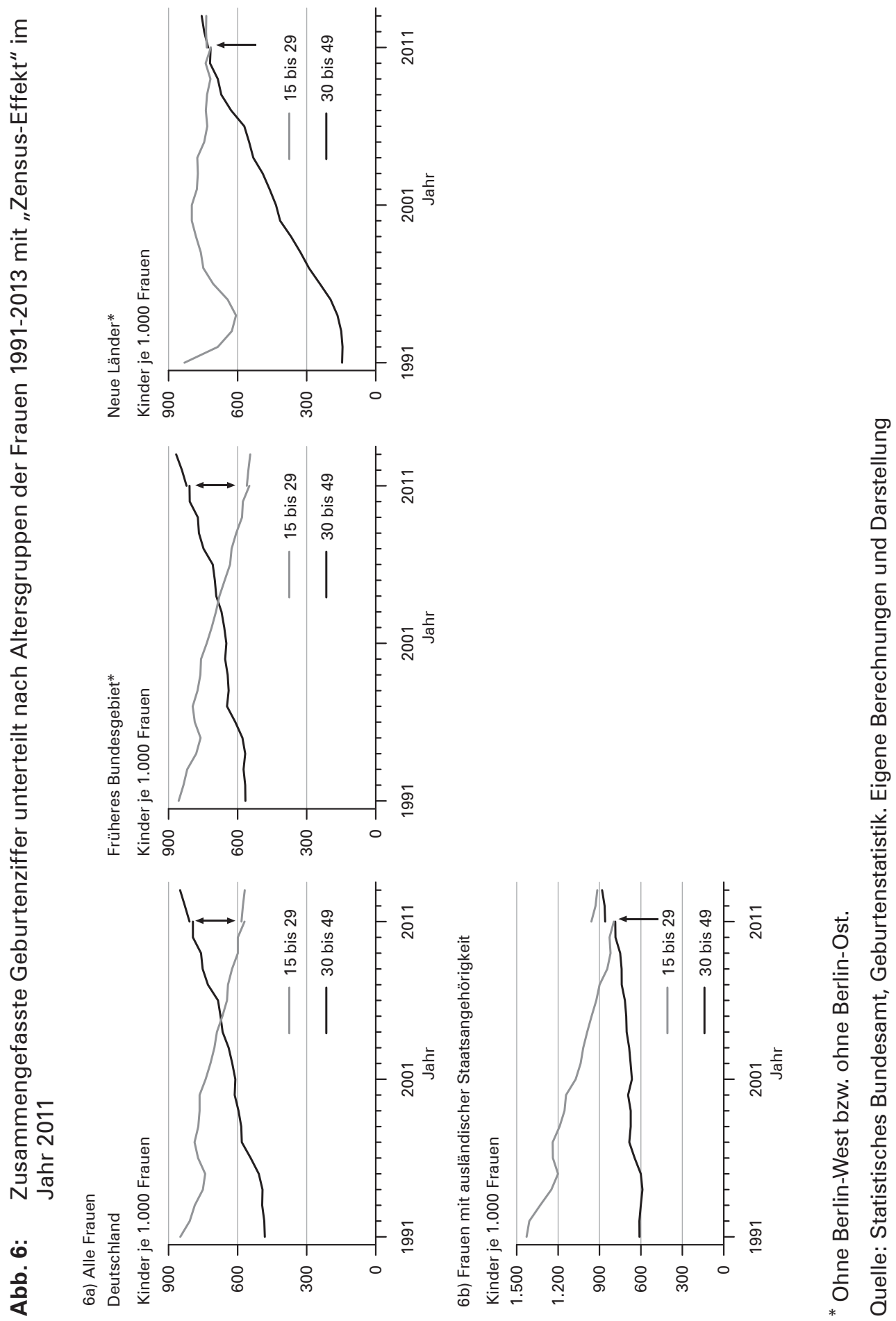


(22 \%). In den Jahren 2012 und 2013 stagnierte die Geburtenrate der Ausländerinnen weiterhin auf dem Niveau von rund 1,80 Kindern je Frau. Der Anteil der Geborenen der ausländischen Mütter an allen geborenen Kindern betrug seit 1996 stabil zwischen $17 \%$ und $18 \%$.

Für die Entwicklung der zusammengefassten Geburtenziffer sind die langfristigen altersspezifischen Trends ausschlaggebend. Diese können im Wesentlichen anhand von nur zwei Altersgruppen dargestellt werden. Wie Abbildung 6a zeigt, geht die Geburtenhäufigkeit der Frauen unter 30 Jahren zurück, während die Fertilität der 30 - bis 49-Jährigen steigt. Für die jüngere Altersgruppe ergibt sich für das Jahr 2011 nach dem Zensus eine leichte Korrektur. Allerdings liegt auch die korrigierte TFR 2011 unter dem Wert für das Jahr 2010. Damit bleibt der Trend in der Geburtenhäufigkeit der 15- bis 29-Jährigen auch nach dem Zensus rückläufig. Die justierte Geburtenziffer bei der älteren Altersgruppe fügt sich in die bisherige Entwicklung nahtlos ein und stört den Verlauf nicht. Dies gilt ebenso für Westdeutschland und mit kleiner Variation der Verläufe - für die neuen Länder.

Bei den Frauen mit ausländischer Staatsangehörigkeit hat die zensusbedingte Korrektur zu einem Sprung im Verlauf der Geburtenhäufigkeit der jüngeren Frauen geführt (Abb. 6b). Die kumulierte Geburtenziffer der 15- bis 29-Jährigen im Jahr 2011 stieg um $20 \%$. Die abnehmende Tendenz bleibt jedoch offenbar weiterhin bestehen. Bei der Altersgruppe der 30- bis 49-Jährigen sind die Korrekturen mit $+9 \%$ vergleichsweise gering. Die Hauptursache für den zu stark eingeschätzten Rückgang der Geburtenrate der Ausländerinnen liegt somit vor allem bei den $\mathrm{Ge}$ burtenziffern der 20- bis 29-Jährigen. Die scheinbar systematische Überzeichnung der Ausländerinnenzahl insbesondere in dieser wanderungsaktiven Altersgruppe führte dazu, dass diese zu niedrig ausgewiesen wurden.

\subsection{Auswirkungen des Zensus auf die Kohortenfertilität}

Die kohortenspezifische durchschnittliche Kinderzahl je Frau ist in Deutschland für die Jahrgänge ab 1930 verfügbar. ${ }^{1}$ Für die Korrektur der altersspezifischen Kohortenziffern nach dem Zensus 2011 wäre eine jahrgangsweise Rückrechnung erforderlich. Allerdings hat sich die Anzahl der Frauen in den Jahrgängen bis Anfang der 1960er Jahre durch den Zensus nur wenig verändert (durchschnittlich -0,9 \%), sodass von einer marginalen Verzerrung der Kohortenwerte ausgegangen werden kann und eine aufwändige und mit großen Unsicherheiten behaftete Rückrechnung nicht angemessen wäre. Bei den Jahrgängen im gebärfähigen Alter, vor allem bei den 1980er und 1990er Kohorten, haben sich die Besetzungszahlen nach dem Zen-

1 Die Kohortenfertilität wird hier nur in Bezug auf alle Frauen betrachtet. Eine separate Analyse für deutsche und ausländische Frauen erscheint vor allem deshalb nicht sinnvoll, weil sich die ausländische Bevölkerung durch Wanderungsbewegungen fortwährend verändert und ihre Kohorten schwer kontrollierbaren exogenen Einflüssen unterworfen sind. Für deutsche Frauen wäre eine kohortenspezifische Betrachtung mit Einschränkung möglich. Da sich diese aber nach dem Zensus kaum verändert hat, würde eine solche Betrachtung keinen Informationsgewinn im Hinblick auf die Kohortenfertilität der Deutschen bringen. 
sus etwas stärker reduziert als bei den älteren Kohorten. Aber auch hier sind die Veränderungen in den einzelnen altersspezifischen Geburtenziffern insgesamt gering, wie in Abschnitt 3.2 bereits gezeigt wurde. Die kumulierten Kohortenziffern der Frauen im fertilen Alter veränderten sich deshalb nach dem Zensus nur leicht.

Eine weitere Möglichkeit, die Zensuseinflüsse auf die Kohortenfertilität zu überprüfen, bietet der Mikrozensus (Statistisches Bundesamt 2013). Die zwei Fragen nach den geborenen Kindern werden im Mikrozensus alle vier Jahre gestellt. Die letzte Befragung fand im Jahr 2012 statt. Die bisher veröffentlichten Ergebnisse des Mikrozensus 2012 beruhten auf der alten Hochrechnung, welche die Zensuskorrekturen noch nicht berücksichtigte. Die Ergebnisse aus der neuen zensusjustierten Hochrechnung liegen nun ebenfalls vor und ermöglichen einen Vergleich mit den Resultaten aus der alten Hochrechnung. Im Folgenden werden diese für die durchschnittliche Kinderzahl je Frau (siehe Abschnitt 2) und für die Verteilung der Frauenjahrgänge nach Zahl der geborenen Kinder (Parität) gegenübergestellt.

Die Entwicklung der durchschnittlichen Kinderzahl je Frau ist in Abbildung 7 dargestellt. Für die Jahrgänge 1963 und älter ist diese endgültig, für die jüngeren Kohorten ist die bis zum Jahr 2012 erreichte Kinderzahl abgebildet. Die Unterschiede zwischen den Ergebnissen nach der alten (gepunktete Linie) und nach der neuen (durchgezogene Linie) Hochrechnung sind optisch kaum wahrnehmbar. Die absoluten Differenzen sind, wie aus Abbildung 8 ersichtlich, gering. Am höchsten sind die Abweichungen mit knapp 30 Kindern je 1000 Frauen in den Kohorten 1976 bis 1981.

Bei den westdeutschen Frauenkohorten sind die Differenzen ähnlich wie für Frauen in Deutschland insgesamt. In den neuen Ländern ist die durchschnittliche Kinderzahl bei den jungen Kohorten nach dem Zensus etwas stärker gestiegen. In den Jahrgängen 1976 bis 1983 betragen die Abweichungen 40 bis 60 Kinder je 1000 Frauen. Allerdings sind diese Unterschiede kleiner als der zweifache absolute Standardfehler und befinden sich somit innerhalb des $95 \%$-igen Konfidenzintervalls (Statistisches Bundesamt 2013: 66). Bei den älteren Kohorten ab 1964 sind dagegen die Abweichungen bei den ostdeutschen Frauen vernachlässigbar gering.

Hinter der durchschnittlichen Kinderzahl steht die Struktur der Frauen eines Jahrgangs nach der Zahl der geborenen Kinder, die sogenannte Paritätsverteilung. Die Interaktion zwischen den Paritäten beeinflusst die endgültige durchschnittliche Kinderzahl. Durch die neue Hochrechnung unter Berücksichtigung der Zensusergebnisse hat sich die Paritätsverteilung nur geringfügig verändert. Tabelle 3 zeigt im oberen Teil die prozentuale Paritätsverteilung der Kohortengruppen nach der neuen Hochrechnung unter Berücksichtigung der zensusjustierten Bevölkerung. Im unteren Teil der Tabelle sind die Differenzen zu den Ergebnissen der "alten“ Hochrechnung dargestellt. Diese zeigen tendenziell einen etwas kleineren Kinderlosenanteil und etwas höhere Anteile der Mütter mit einem und zwei Kindern. Die einzelnen Differenzen sind allerdings sehr gering und verändern nur unwesentlich die bisherigen Erkenntnisse über die Paritätsverteilung der Frauenkohorten. 
Abb. 7: Endgültige bzw. erreichte Kinderzahl je Frau nach Geburtskohorten Ergebnissen des Mikrozensus 2012 nach der neuen (nach Zensus) und nach der alten (vor Zensus) Hochrechnung

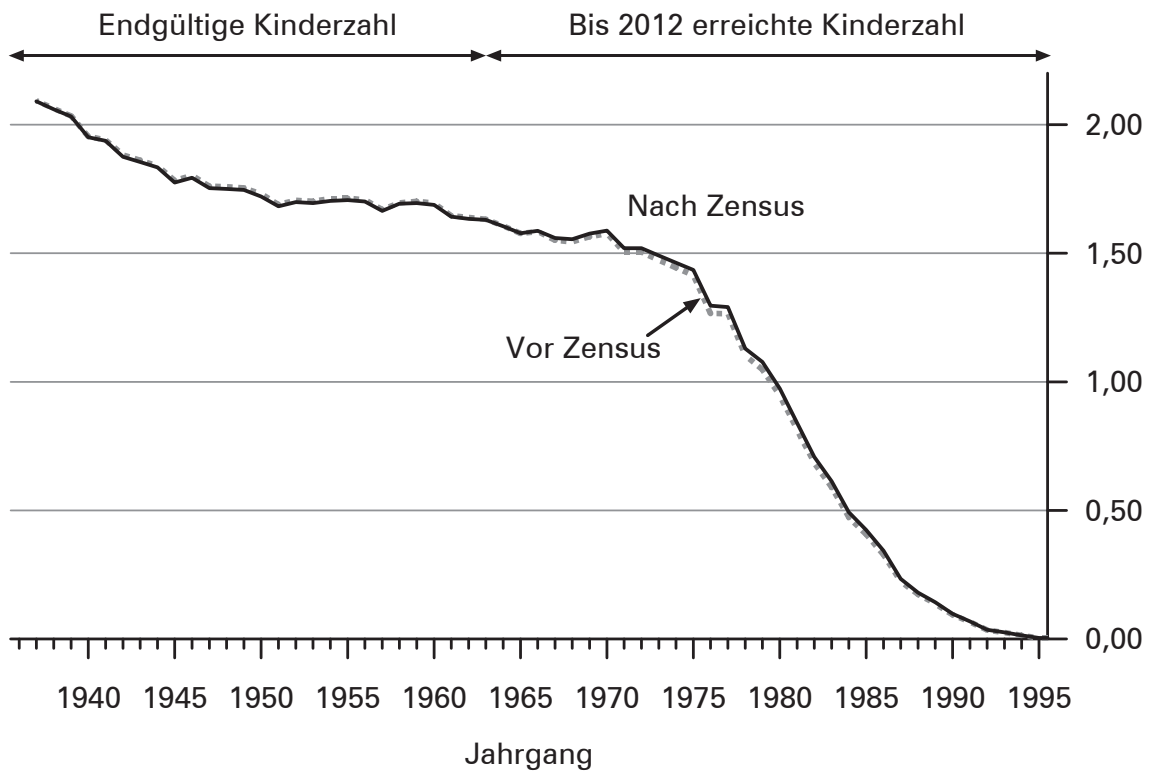

Quelle: Statistisches Bundesamt, Mikrozensus 2012. Eigene Berechnungen und Darstellung

Abb. 8: Absolute Differenzen in der endgültigen bzw. erreichten Kinderzahl je Frau nach Geburtskohorten zwischen den Ergebnissen des Mikrozensus 2012 nach der neuen (nach Zensus) und nach der alten (vor Zensus) Hochrechnung

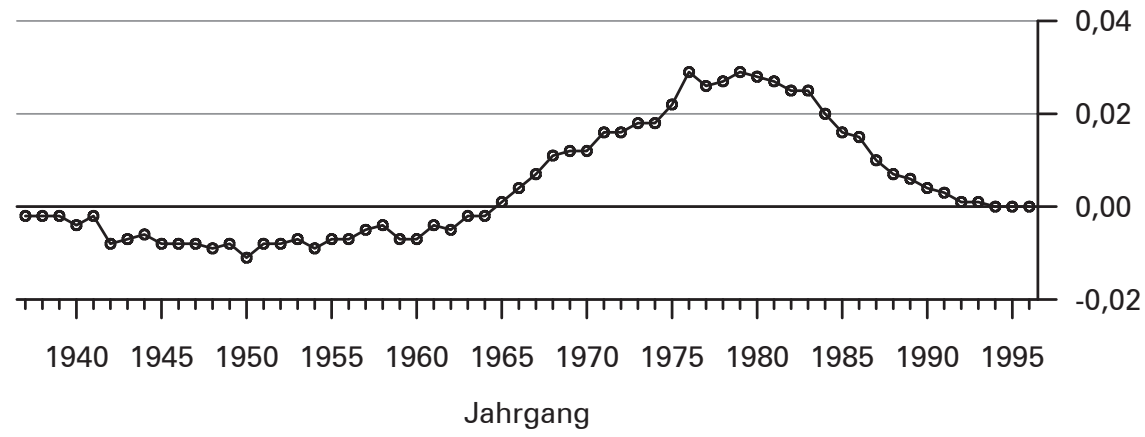

Quelle: Statistisches Bundesamt, Mikrozensus 2012. Eigene Berechnungen und Darstellung 


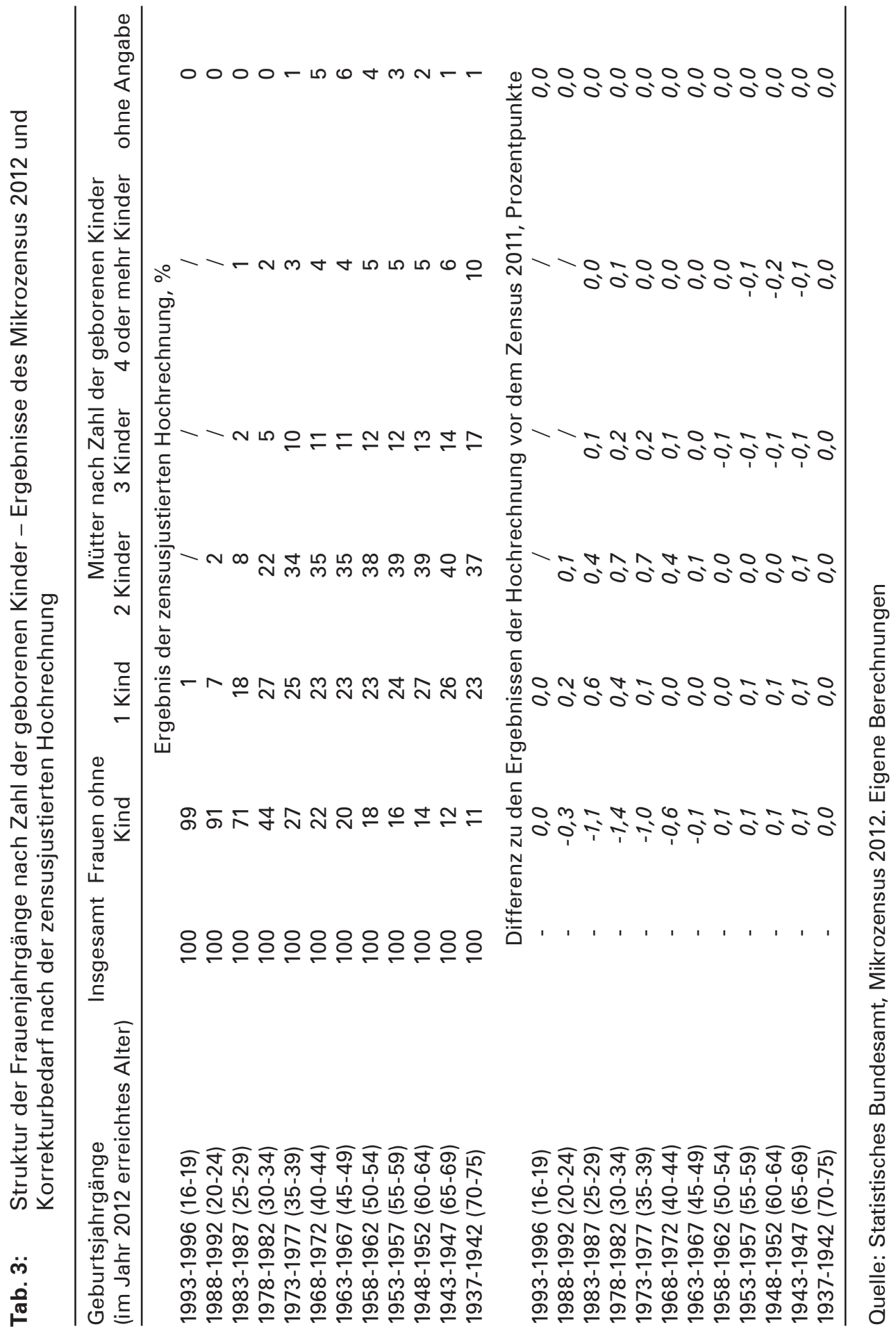




\subsection{Zwischenfazit}

Der Zensus 2011 ergab eine um 321.000 geringere weibliche Bevölkerung im gebärfähigen Alter als die laufende Bevölkerungsfortschreibung. Dadurch haben sich die Geburtenmaßzahlen, die sich auf die Anzahl der Frauen beziehen oder auf den hochgerechneten Stichprobenergebnissen des Mikrozensus 2012 beruhen, insgesamt nur leicht verändert. Die zusammengefasste Geburtenziffer des Jahres 2011 nahm von 1,36 auf 1,39 Kinder je Frau um rund $2 \%$ zu. Bei den langfristigen altersspezifischen Trends lag die zensusbedingte Korrektur im Bereich der durchschnittlichen jährlichen Schwankungen. Auch die Auswirkungen des Zensus 2011 auf die Kohortenfertilität waren gering. Auf die Geburtenziffern nach der Staatsangehörigkeit der Frau hat sich die zensusbedingte Justierung unterschiedlich ausgewirkt. Während die zusammengefasste Geburtenziffer der Frauen mit deutscher Staatsangehörigkeit mit 1,34 Kindern je Frau beinahe unverändert blieb, stieg sie bei den ausländischen Frauen von 1,58 auf 1,82 Kinder je Frau deutlich. Diese Korrektur erfordert eine Überprüfung der bisherigen Befunde zur Fertilität der ausländischen Frauen. Auf die Fertilitätstrends insgesamt war aber der Einfluss der Ausländerinnen bisher eher gering.

\section{$4 \quad$ Entwicklung der Kohortenfertilität in Deutschland}

Der Vergleich der wichtigsten Indikatoren hat gezeigt, dass das demografische Bild der Geburtenentwicklung nach dem Zensus 2011 im Wesentlichen erhalten geblieben ist. Die Untersuchungen der Fertilität können damit ohne große Zäsuren fortgeführt werden. Was macht aber dieses Bild im Kern aus? Im Folgenden versuchen wir uns dieser Frage zu nähern, indem wir die Analyseergebnisse auf Basis der Daten der laufenden Geburtenstatistik durch die Ergebnisse des Mikrozensus 2012 ergänzen. In einen Zusammenhang gestellt erlauben diese Befunde einen Einblick in die wesentlichen Züge des Geburtenverhaltens und einen Ausblick auf die künftige Fertilitätsentwicklung.

\subsection{Dauerhafter Anstieg der Kohortenfertilität derzeit nicht absehbar}

Die in den 1930er Jahren geborenen Frauen - zum Großteil die Mütter der BabyBoom-Generation - haben durchschnittlich mehr als zwei Kinder geboren (Abb. 9). Ihre Familiengründungsphase fiel in die Zeit des wirtschaftlichen Aufschwungs der 1950 er und 1960er Jahre. Bereits innerhalb der 1930er Jahrgänge zeichnete sich jedoch ab, dass die endgültige Kinderzahl kontinuierlich abnimmt. Besonders schnell sank sie aufgrund des Rückgangs bei höheren Paritäten - 4 oder mehr Kindern - zwischen den Kohorten 1934 und 1944 (Statistisches Bundesamt 2009, 2013). Anschließend hat sich die Paritätsverteilung der Mütter weitgehend stabilisiert, die Kinderlosigkeit nahm aber kontinuierlich zu. Dies führt bis heute zu einem weniger rasanten, jedoch beständigen Rückgang der Kohortenfertilität. Der jüngste Jahrgang, der das 
Abb. 9: Endgültige sowie bis zum Alter von 29 erreichte durchschnittliche Kinderzahl nach Frauenkohorten

Geborene je Frau

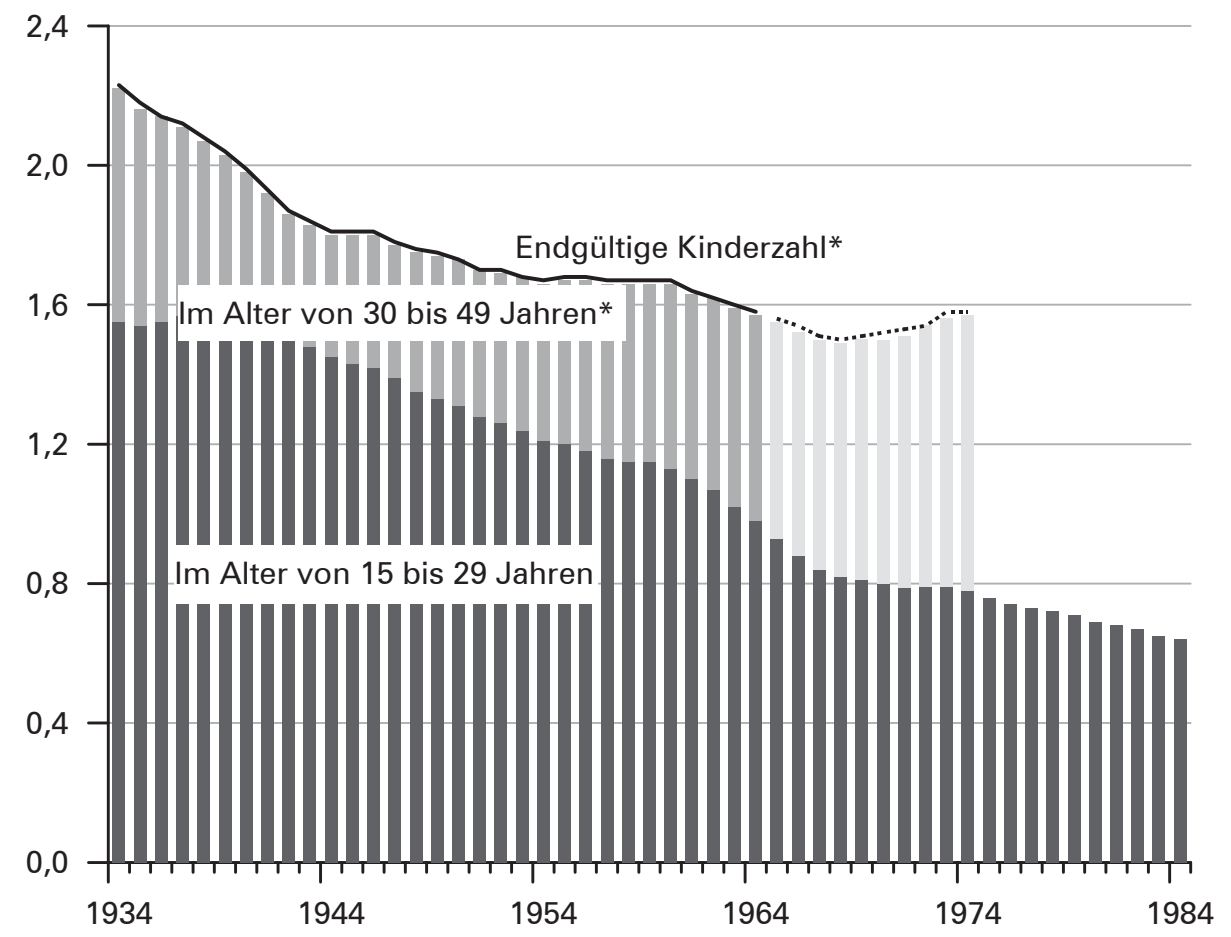

* Für die Jahrgänge 1965 bis 1974 geschätzte Werte.

Quelle: Statistisches Bundesamt 2013, Geburtenstatistik. Eigene Berechnungen und Darstellung

fünfzigste Lebensjahr erreichte und dessen kumulierte Geburtenziffer statistisch als endgültig gilt, ist der Jahrgang 1964 mit insgesamt 1,57 Kindern je Frau.

Es ist bereits absehbar, dass die endgültige Kinderzahl der späten 1960er Jahrgänge weiter auf circa 1,49 Kinder je Frau sinken wird. Danach ist eine leichte Erholung der abgeschlossenen Kohortenfertilität zu erwarten (Pötzsch 2010a; Goldstein) Kreyenfeld 2011; Sobotka 2011). Zu dieser Erholung trägt einerseits bei, dass sich die kumulierte Kohortenfertilität bis zum Alter von 29 Jahren bei den Jahrgängen 1969 bis 1973 stabilisierte. Andererseits stieg aber die Geburtenhäufigkeit dieser Kohorten im Alter ab 30 Jahren kontinuierlich weiter. Zusammen haben diese beiden Effekte für eine günstige Konstellation gesorgt, die sich jedoch ab dem Jahrgang 1974 nicht mehr fortsetzt. Zwischen den Jahrgängen 1974 und 1984 nahm die kumulierte Fertilität bis zum Alter von 29 Jahren wieder kontinuierlich ab. Wie sie sich im höheren Alter entwickeln wird, ist zwar noch offen, da diese Jahrgänge derzeit das Ende der gebärfähigen Phase noch nicht erreicht haben. Einige Hinweise darauf können allerdings aus der Analyse des Aufschubs und des Nachholens der Geburten, des 
sogenannten Postponement \& Recuperation-Prozesses (P\&R-Prozess), gewonnen werden (Frejka/Calot 2001; Sobotka et al. 2011; Frejka 2012; Pötzsch 2013).

Der Ausgangspunkt der P\&R-Analyse ist das Verständnis der Kohortenfertilität als ein Prozess, bei dem die frühere Fertilitätsbiografie der Frauen ihre künftigen Fertilitätsentscheidungen maßgeblich beeinflusst. Jede Frauenkohorte hat ihr eigenes Geburtentiming, das sich aus dem für sie typischen Geburtenverhalten ergibt. Bekommen z.B. die meisten Frauen eines Jahrgangs Kinder tendenziell in der jüngeren gebärfähigen Phase, nimmt die kumulierte durchschnittliche Kinderzahl schnell zu, erreicht dann relativ bald ihren Höhepunkt und bleibt anschließend beinahe konstant (Abb. 10: Jahrgang 1944). Entscheiden sich Frauen eines Jahrgangs dagegen mehrheitlich später für Kinder, steigt die kumulierte Geburtenzahl langsamer (Jahrgang 1962). Ob die endgültige Kinderzahl dann schließlich kleiner ausfällt als bei den Kohorten mit einem relativ „jungen“ Geburtentiming, hängt davon ab, ob im jungen Alter nicht realisierte Geburten später im vollen Umfang nachgeholt werden. In Deutschland hat das Verschieben des Timings auf das höhere Alter bisher tendenziell eine niedrigere endgültige Kinderzahl nach sich gezogen (Pötzsch 2013).

In Relation zum Jahrgang 1944 hat die Kohorte $1962^{2}$ ihre Geburten später realisiert. Der größte Abstand in der kumulierten Fertilität wurde im Alter von Ende

Abb. 10: Kumulierte altersspezifische Geburtenziffern der ausgewählten Frauenkohorten

Geborene je 1000 Frauen

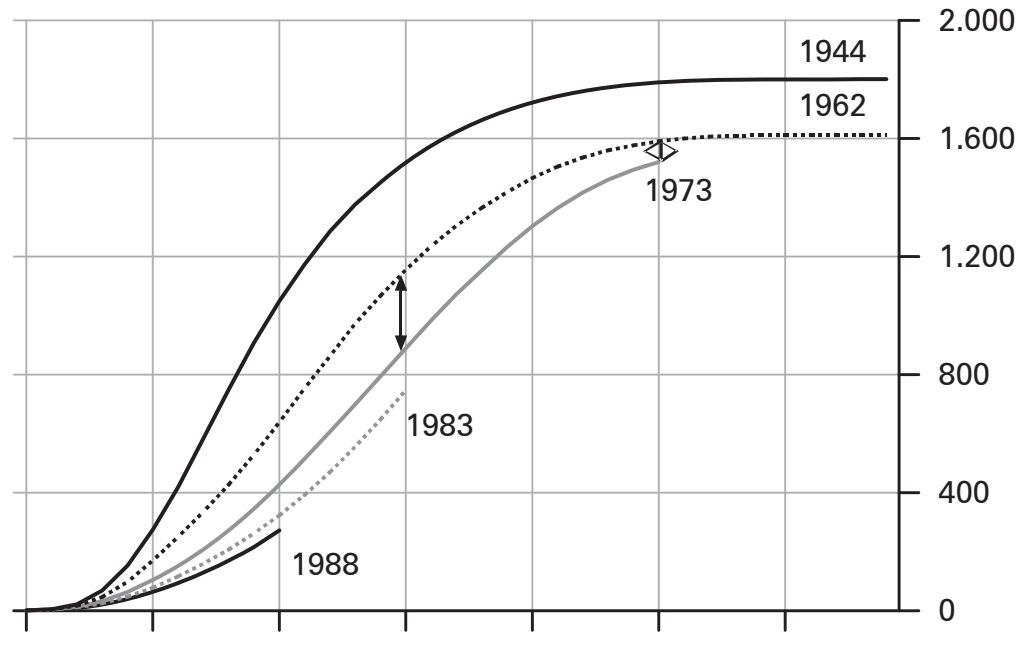

$\begin{array}{lllllll}15 & 20 & 25 & 30 & 35 & 40 & 45\end{array}$

Alter im Jahr 2013

Quelle: Statistisches Bundesamt, Geburtenstatistik. Eigene Berechnungen und Darstellung

2 Die Kohorte 1962 wird für weitere Vergleiche als Referenz herangezogen, da dies der vorübergehend letzte abgeschlossene Jahrgang mit der endgültigen Kinderzahl von gut 1,6 ist, danach wird die endgültige Kinderzahl aller Voraussicht nach unter 1,6 sinken. 
zwanzig erreicht. Anschließend hat sich der Abstand zwischen beiden Kurven zwar reduziert, der Nachholumfang hat aber nicht ausgereicht, um den Rückgang der Fertilität im jungen Alter zu kompensieren, und die CTFR fiel um 0,2 kleiner aus. Die 1973 geborenen Frauen haben bis zum Alter von 28 Jahren durchschnittlich noch weniger Kinder geboren als die Frauen der Kohorte 1962 mit einer endgültigen Kinderzahl je Frau von 1,61. Ab dem Alter von 29 Jahren holen sie nun die "aufgeschobenen" Geburten auf. Der Abstand zwischen den beiden Kurven hat sich bis zum Alter von 40 Jahren um drei Viertel reduziert und betrug 70 Geburten je 1000 Frauen. Diese Lücke wird im Alter ab 41 Jahre voraussichtlich nicht vollständig geschlossen werden können, sie wird sich aber beträchtlich reduzieren (kumulierte Kohortenfertilität im Alter von 41 bis 49 Jahren beträgt derzeit ca. 35 Geburten je 1000 Frauen). Die endgültige Kinderzahl je Frau der Kohorte 1973 wird schätzungsweise 1,57 betragen. Die noch jüngeren Jahrgänge 1983 und 1988 repräsentieren die aktuellen Tendenzen in der Kohortenfertilität. Es wird dabei deutlich, dass immer weniger Geburten in der jüngeren fertilen Phase realisiert werden.

Um Auswirkungen des jüngsten Geburtenaufschubs auf die endgültige Kinderzahl bewerten zu können, versuchen wir das künftige Nachholpotenzial mit Hilfe einer grafischen Darstellung einzuschätzen (Abb. 11). Für diesen Zweck definieren wir die Kohorte 1962 mit der CTFR von 1,61 als eine Referenzkohorte. Für jede weitere Kohorte wird eine Differenz zwischen den kumulierten CFR dieser Kohorte und der Referenzkohorte 1962 gebildet. Da die Geburtenhäufigkeit von Kohorte zu Kohorte abnimmt, sind diese Abweichungen negativ. Sie verstärken sich in der Regel bis zum Alter von 29 Jahren, welches den sogenannten "Tiefpunkt" darstellt, und vermindern sich anschließend, wie bereits am Beispiel der Kohorten 1962 und 1973 in Abbildung 10 gezeigt wurde. Die Phase bis zum Tiefpunkt entspricht dem Geburtenaufschub; nach dem Tiefpunkt beginnt das Nachholen der Geburten. Um den Aufschub der Geburten mit dem Nachholumfang zu vergleichen, werden je Kohorte die altersspezifischen Geburtenziffern jeweils in der Aufschub- und in der Nachholphase addiert. Die Aufschubphase erstreckt sich von 15 bis 29 Jahren. Für die Nachholphase werden mehrere Altersgrenzen gewählt, damit auch für die noch relativ jungen Jahrgänge Aussagen getroffen werden können: von 30 bis 35, von 30 bis 37 sowie von 30 bis 40 Jahren. Für diese Phasen betrachten wir die Abweichungen in den jeweiligen kumulierten Geburtenziffern der Kohorten 1963 bis 1984 von der Referenzkohorte 1962 (Abb. 11 oben).

Erwartungsgemäß sind die Abweichungen in der Aufschubphase bis zum Alter von 29 Jahren negativ, während sie in der Nachholphase positiv sind, da die Kohortenfertilität der jüngeren Jahrgänge im Alter ab 30 Jahre zunimmt. Hier finden sich die bereits beschriebenen Entwicklungen in einer neuen Darstellungsform wieder: Der Rückgang der Kohortenfertilität im jungen fertilen Alter war bei den 1960er Jahrgängen besonders stark. Trotz zunehmender Fertilität im Alter ab 30 Jahre kann er bis zum Ende der fertilen Phase kaum noch kompensiert werden. Vielmehr hat sich die Restabweichung im Alter 40 Jahre bis zum Jahrgang 1968 vergrößert, was auf ein absehbares Sinken der endgültigen Kinderzahl hinweist (Abb. 11 unten).

Für die Jahrgänge 1969 und 1973 verdeutlicht diese Darstellung die bereits beschriebene günstige Konstellation aus der Verstetigung der kumulierten CFR im Al- 
Abb. 11: Absolute Veränderung der altersspezifischen Kohortenfertilität (cohort fertility rate, CFR) in der Aufschub- und Nachholphase im Vergleich zur Kohorte 1962 (CTFR`1962 = 1,61 Kinder je Frau)

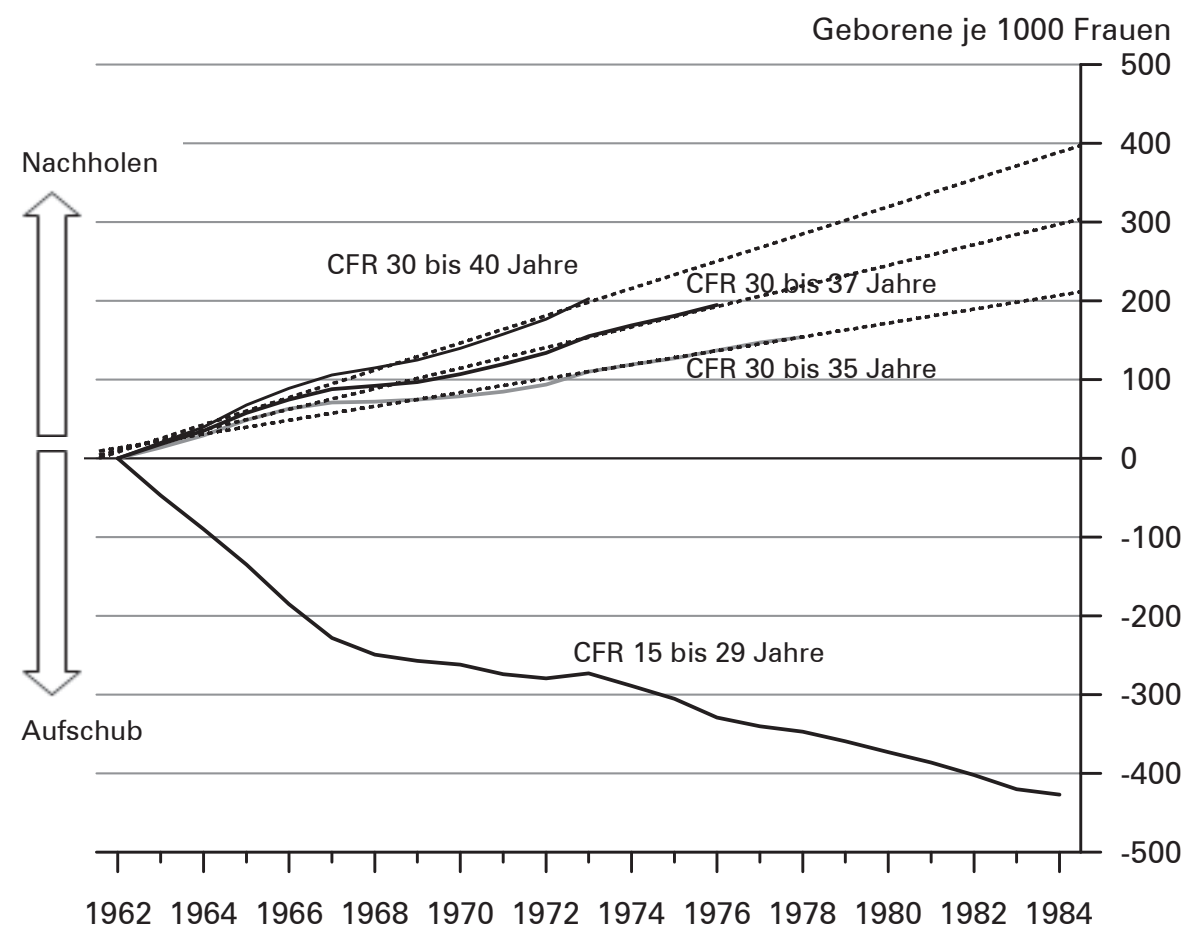

Restabweichung im Alter 40 Jahre. Ab Jahrgang 1974 hypothetische Werte bei formaler Fortschreibung des Nachholtrends

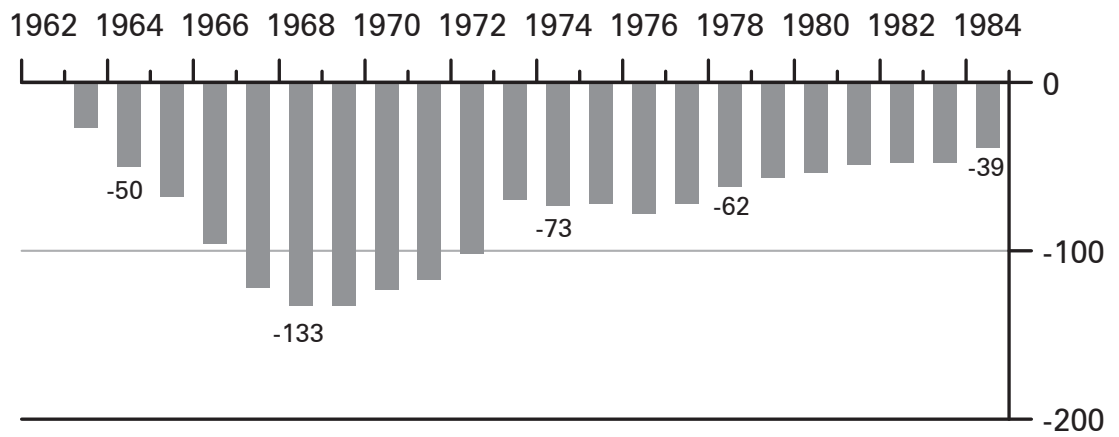

Anmerkungen: Die CFR $x$ bis $(x+n)$ Jahre ist hier die Summe der altersspezifischen Geburtenziffern einer Kohorte in den entsprechenden Altersjahren. Die absolute Veränderung wurde als Differenz zwischen der CRF einer Kohorte c und der CFR der Kohorte 1962 für die jeweilige Altersspanne berechnet. Die gepunktete Linie bildet den linearen Trend ab.

Quelle: Statistisches Bundesamt, Geburtenstatistik. Eigene Berechnungen und Darstellung 
ter unter 30 Jahren einerseits und des verstärkten Nachholens im Alter zwischen 30 und 40 Jahren andererseits. Dadurch wird die endgültige Kinderzahl leicht steigen. Ab der Kohorte 1974 sinkt die Fertilität im Alter unter 30 Jahren kontinuierlich, was eine erneute Zunahme des Geburtenaufschubs bedeutet. Wie würde sich dieser Rückgang auf die endgültige Kinderzahl auswirken?

Mit Hilfe von Abbildung 11 (oben) kann nachvollzogen werden, welcher Nachholumfang in einzelnen Altersstufen erforderlich wäre, um den Fertilitätsrückgang im Alter unter 30 Jahren zu kompensieren und mittelfristig die endgültige Kinderzahl von 1,6 zu erreichen. Dieses Ziel würde dann frühestens ab der Kohorte 1980 annähernd erreicht werden können, wenn sich die Nachholtrends der letzten 16 Jahre linear fortsetzen würden (vgl. gestrichelte Trendlinien). Dieses Szenario scheint auf den ersten Blick nicht unrealistisch. Bei näherer Betrachtung wird jedoch deutlich, dass dies eine größere Herausforderung darstellt. Zum einen zeichnet sich insbesondere in Westdeutschland ab, dass das Nachholen bis zum Alter von 35 Jahren zur Stagnation neigt. Die hier vorgenommene ungebremste Trendextrapolation ist somit bereits eine optimistische Annahme. Zum anderen würde eine solche Verlagerung der Kohortenfertilität ins höhere gebärfähige Alter bedeuten, dass die Fertilität im Alter von 36 bis 40 Jahren zwischen den Kohorten 1962 und 1984 um 250 \% steigen und das Nachholen im Alter ab 36 Jahre im gleichen Umfang stattfinden müsste, wie in der starken fertilen Phase zwischen 30 und 35 Jahren.

Damit wäre allein für die mittelfristige Stabilisierung der endgültigen Kinderzahl auf dem relativ niedrigen Niveau zwischen 1,5 und 1,6 Kindern je Frau erforderlich, dass die Geburtenhäufigkeit der späten 1970er und 1980er Kohorten im Alter ab 30 Jahre kontinuierlich steigt. Im Hinblick auf die Parität bedeutet dies sowohl mehr erste Geburten im höheren fertilen Alter als auch eine stabile Verteilung der Mütter nach Zahl der Kinder trotz späterer Familiengründung. Denn die endgültige Kinderzahl von 1,6 Kindern je Frau kommt nur dann zustande, wenn circa $80 \%$ der Frauen eines Jahrgangs im Durchschnitt 2 Kinder zur Welt bringen würden. Im Folgenden eruieren wir, inwieweit diese Voraussetzungen erfüllt werden können.

\subsection{Stabilität der Kinderzahl je Mutter ist fragil}

Die durchschnittliche Kinderzahl je Mutter nahm zwischen den 1930er und 1940er Jahrgängen von 2,3 auf 2,0 ab und stabilisierte sich anschließend auf diesem Niveau. Während der Rückgangsphase hat sich der Anteil der Mütter mit vier oder mehr Kindern von $12 \%$ auf $6 \%$ halbiert. Zwischen den späten 1940er und frühen 1970 er Jahrgängen wies die Paritätsverteilung der Mütter nur geringe Schwankungen auf. Die 45 bis 49 Jahre alten Mütter hatten 2012 zu $31 \%$ „nur” ein Kind, zu $48 \%$ zwei Kinder, zu $15 \%$ drei Kinder und zu $6 \%$ vier oder mehr Kinder. Im Durchschnitt bekamen die Mütter im Laufe ihres Lebens 2 Kinder (Statistisches Bundesamt 2015c).

Die Stabilität der Paritätsverteilung der Mütter ist allerdings bei den jüngeren Jahrgängen angesichts des langfristigen Trends zum höheren Erstgeburtsalter gefährdet. Das liegt vor allem daran, dass die Gruppe der Mütter, die vor ihrem 30. Geburtstag eine Familie gründen, immer kleiner wird. Gerade diese Mütter stellten 
aber bisher das Potenzial für Kinderreiche. Für die Frauen der 1960er und 1970er Kohorten zeigten die Mikrozensusergebnisse, dass je mehr Kinder eine Frau im Laufe ihres Lebens zur Welt gebracht hat, desto jünger war sie bei der Geburt ihres ersten Kindes (Statistisches Bundesamt 2015: 30). Die Mütter von Einzelkindern bekamen ihr Kind durchschnittlich im Alter von 30 Jahren. Die Mütter von zwei Kindern waren mit 27 Jahren bei der Familiengründung bereits drei Jahre jünger. Familien mit drei oder mehr Kindern wurden im Durchschnitt von Frauen bis zum Alter von 25 Jahren gegründet. ${ }^{3}$ Eine künftig stabile Paritätsverteilung setzt also voraus, dass sich das Geburtenverhalten im höheren fertilen Alter ändert und der Anteil der Mütter zunimmt, die eine Familie in ihren Dreißigern gründen und anschließend trotzdem noch drei oder mehr Kinder bekommen.

Da das biologische Fenster begrenzt ist, müssten sich dabei auch die Abstände zwischen den Geburten einer Mutter zunehmend verringern. Dies bestätigen auch die Ergebnisse des Mikrozensus: Umso älter eine Mutter mit mindestens drei Kindern zum Zeitpunkt der ersten Geburt war, desto kürzer waren die Abstände zwischen ihren Geburten. Bei Frauen, die im Alter von 20 Jahren erstmals Mutter geworden sind, betrug das Intervall zwischen dem ersten und dritten Kind knapp 10 Jahre (Abb. 12). Bei Frauen, die ihr erstes Kind erst im Alter von 33 Jahren bekommen haben, war dieses Intervall mit 5 Jahren nur halb so groß. Im Durchschnitt vergingen zwischen der ersten und dritten Geburt 7,4 Jahre.

Da immer mehr Frauen erst mit Anfang 30 erstmals Mutter werden und bei Geburt von mehreren Kindern folglich kürzere Geburtenabstände haben, müssten sich tendenziell auch die mittleren Intervalle verkürzen. Jedoch liefert dazu die Statistik der Geburtenabstände zum vorangegangenen Kind der Mutter bei der zweiten, dritten oder weiteren Geburt bisher keinen empirischen Beleg. Die seit 2009 verfügbaren Angaben über die vollständige Geburtenfolge der Mutter zeigen vielmehr, dass die mittleren Abstände zwischen den Geburten von 2009 bis 2014 trotz des sich fortsetzenden Aufschubs der Familiengründung unverändert geblieben sind. „In 50 \% der Fälle kommt ein zweites Kind innerhalb von 3,3 Jahren nach dem Erstgeborenen zur Welt. Die anderen 50 \% der zweitgeborenen Kinder haben einen größeren Abstand zum älteren Geschwisterkind. Die dritten Kinder folgen in der Regel mit einem noch größeren zeitlichen Intervall nach der Geburt des zweiten Kindes. Die Hälfte aller dritten Geburten ereignet sich innerhalb von 3,9 Jahren, die andere Hälfte erfolgt noch später." (Statistisches Bundesamt 2014). Diese Ergebnisse bestätigen zudem den Befund aus dem Mikrozensus 2012, dass das Intervall zwischen der ersten und dritten Geburt im Mittel gut 7 Jahre beträgt. Daraus folgt, dass die Mütter der dritten und weiteren Kinder nach wie vor überwiegend zu sogenannten "frühen Familiengründerinnen“ gehören. Der Anteil derjenigen Frauen, die relativ spät ihr erstes Kind bekommen haben, ist unter den Müttern beim dritten und weite-

3 Diese Mikrozensusergebnisse beziehen sich auf Mütter, die zum Zeitpunkt der Mikrozensusbefragung im Jahr 2012 im Alter von 35 bis 44 Jahren waren und mit ihren Kindern in einem Haushalt lebten. Das so ermittelte durchschnittliche Erstgeburtsalter dürfte tendenziell etwas überhöht sein, da in einigen Fällen das älteste Kind im Haushalt der Mutter bereits fehlen könnte (Quelle: Mikrozensus 2012, eigene Berechnungen). 
Abb. 12: Durchschnittliches Intervall zwischen der ersten und der dritten Geburt nach dem Alter der Mutter beim ersten Kind, ${ }^{*}$ in Jahren

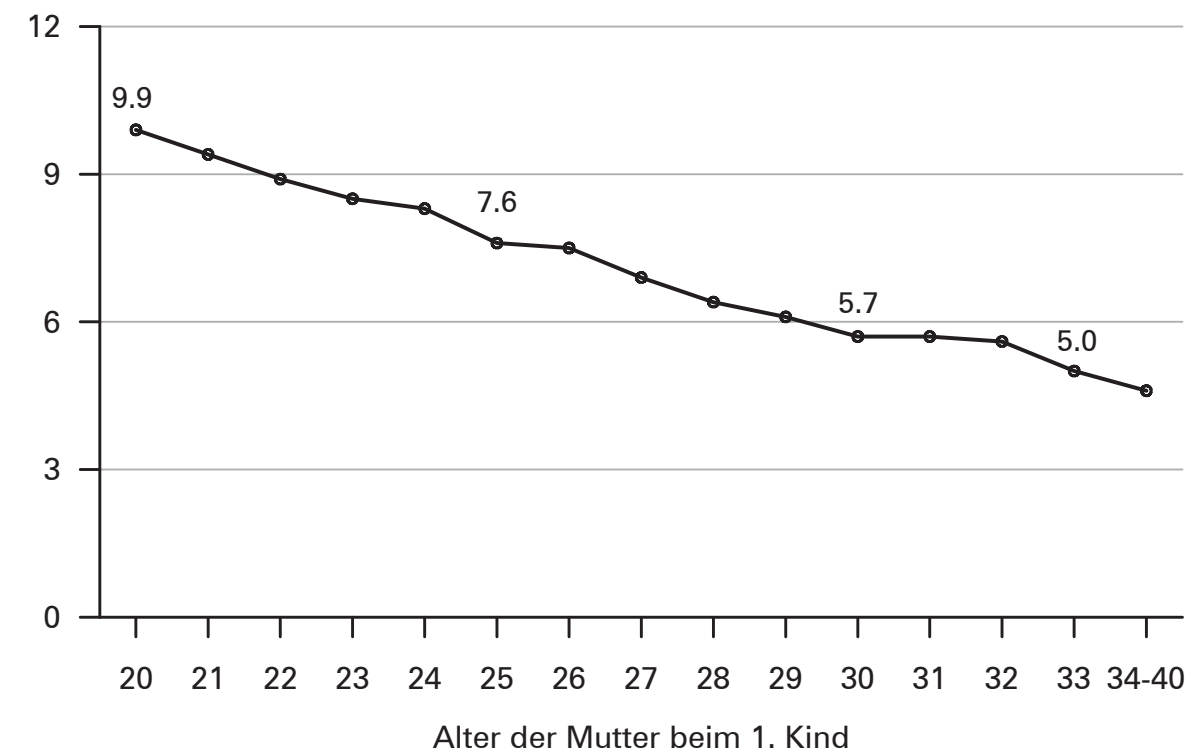

* Einbezogen sind 35- bis 44-jährige Mütter mit mindestens drei Kindern, die zusammen in einem Haushalt leben.

Quelle: Statistisches Bundesamt, Mikrozensus 2012 (zensusjustierte Hochrechnung). Eigene Berechnungen und Darstellung

ren Kind offenbar relativ gering. Deshalb wird die Verteilung der Geburtenabstände zum vorangegangenen Kind von den kürzeren Intervallen zwischen ihren Geburten kaum beeinflusst.

Die Entwicklung der Intervalle zwischen den Geburten ist ein wichtiger Frühindikator dafür, ob nicht nur die ersten und zweiten, sondern auch weitere Geburten "nachgeholt" werden. Wie aus Abb. 13 ersichtlich, betrug der Anteil der zweiten bzw. dritten Kinder, die innerhalb von 2 Jahren nach dem vorangegangenen Kind der Mutter zur Welt gekommen sind, im Zeitraum 2009 bis 2013 stabil etwa $16 \%$ bis $18 \%$. Mit einem Abstand bis unter 3 Jahren wurden etwa $44 \%$ der zweiten bzw. ca. $36 \%$ der dritten Kinder geboren. Es ist außerdem bemerkenswert, dass der Anteil der zweiten Kinder mit einem Abstand zum Erstgeborenen von 2 bis unter 3 Jahren fast genau so groß war wie der Anteil der zweiten Kinder, die erst nach einer Pause von fünf und mehr Jahren zur Welt gekommen waren. Bis zur dritten Geburt waren die Abstände tendenziell noch größer: 37 \% der dritten Kinder waren im Jahr 2013 mindestens um fünf Jahre jünger als das vorangegangene Geschwisterkind.

Für das aktuelle Geburtenverhalten ist eine Konstellation aus dem Trend zu immer späterer Familiengründung, den langen Geburtenabständen sowie dem negativen Zusammenhang zwischen dem Zeitpunkt der ersten Geburt einerseits und der Gesamtzahl der geborenen Kinder andererseits charakteristisch. Sollte sich diese 
Konstellation in näherer Zukunft nicht ändern, würde es zum Rückgang des Mütteranteils mit drei oder mehr Kindern kommen.

Abb. 13: Anteil der Geburten, die nach einem entsprechenden Abstand zum Geburtstag des vorangegangenen Kindes erfolgten* (in Prozent)
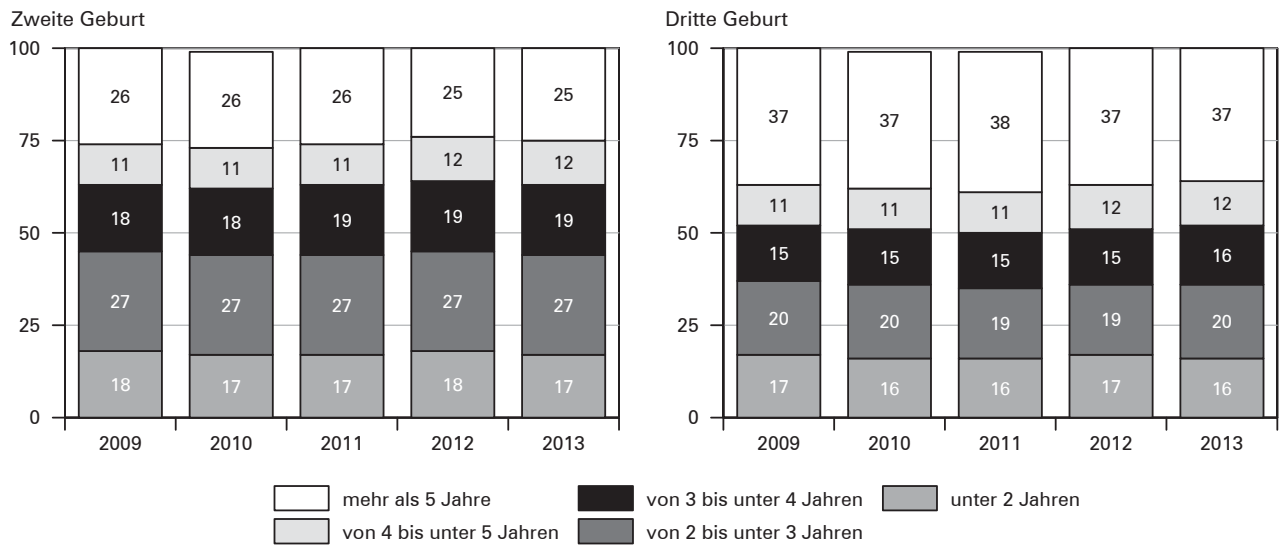

* Bei den Geburten von Zwillingen und weiteren Mehrlingen wurde nur eines der Kinder berücksichtigt.

Quelle: Statistisches Bundesamt, Geburtenstatistik. Eigene Berechnung und Darstellung

\subsection{Trend zu höherer Kinderlosigkeit setzt sich fort}

Der Anstieg der Kinderlosigkeit hat insbesondere in Westdeutschland die Kohortenfertilität der letzten gut zwanzig Jahre stark beeinflusst (Statistisches Bundesamt 2013). Nach den Berechnungen von Sobotka würde die endgültige Kinderzahl des Jahrgangs 1965 statt 1,5 Kinder je Frau 1,7 betragen, wenn die Kinderlosigkeit auf dem Niveau der Kohorte 1940 geblieben wäre (Sobotka 2011: 273). Auch in den neuen Ländern, wo die Kinderlosigkeit bis in die 1960er Jahrgänge lediglich $11 \%$ betrug und damit nur halb so hoch war wie in Westdeutschland, steigt der Kinderlosenanteil.

Die Kinderlosenquote ändert sich bereits ab dem Alter von 41 Jahren kaum noch. Die Zahl der über 40-Jährigen, die ein zweites oder weiteres Kind zur Welt bringen, nimmt zwar zu, ihr erstes Kind bekommen Frauen im Alter von über 40 Jahre jedoch nach wie vor sehr selten. Lediglich $3 \%$ der ersten Kinder wurden 2013 von Frauen im Alter zwischen 40 und 49 Jahren geboren, ab dem Alter von 42 Jahren war es lediglich $1 \%$. Auch ein Vergleich des Kinderlosenanteils an den jeweiligen Frauenkohorten zwischen den Mikrozensusbefragungen 2008 und 2012 zeigt nur marginale Veränderungen bereits ab dem Alter von 39 Jahren. Eine Ausnahme stellen allerdings Frauen mit Hochschulabschlüssen dar, deren Familiengründungsphase länger dauern kann (Statistisches Bundesamt 2013: 22 und 36). 
Abb. 14: Anteil der Frauen ohne Kind an allen Frauen der Geburtsjahrgänge, die im Jahr 2012 zwischen 41* und 69 Jahren alt waren (in Prozent)

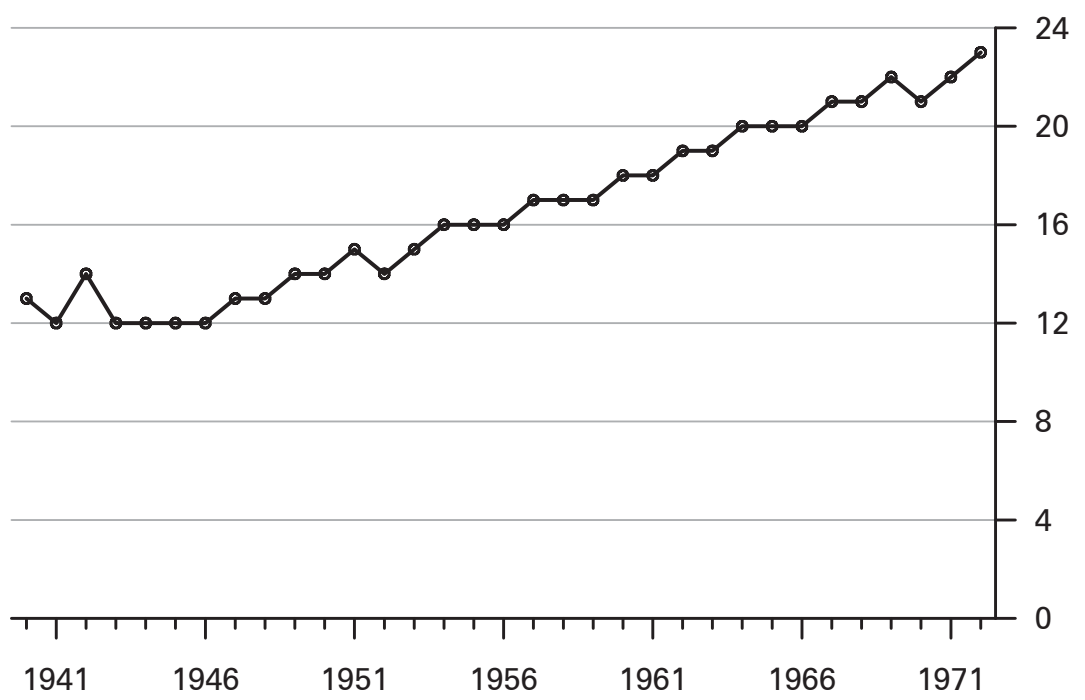

* Ab dem Alter von 41 Jahren verändert sich die Kinderlosenquote nur marginal.

Quelle: Statistisches Bundesamt, Mikrozensus 2012 (zensusjustierte Hochrechnung). Eigene Berechnungen und Darstellung

In der Kohorte 1971, die im Jahr 2012 das Alter von 41 Jahren erreicht hat, sind $22 \%$ der Frauen kinderlos geblieben. Die Kinderlosenquote war damit um 10 Prozentpunkte höher als in den Kohorten 1940 bis 1946 (12 \%). Zwischen den Jahrgängen 1946 und 1971 nahm die Kinderlosenquote, abgesehen von einzelnen kleinen Schwankungen, fast linear zu (Abb. 14).

Inwieweit und wie lange die endgültige Kinderlosenquote in Zukunft steigen wird, ist noch offen. Einerseits gibt es positive Impulse, wie zum Beispiel bei den westdeutschen Frauen mit akademischen Bildungsabschlüssen. Ihre endgültige Kinderlosenquote hat sich zuletzt bei $29 \%$ (nach der zensusjustierten Hochrechnung) stabilisiert und wird in den nächsten Jahren voraussichtlich sogar leicht sinken (Statistisches Bundesamt 2013: 37; Statistisches Bundesamt 2015c). Andererseits deuten einige Befunde darauf hin, dass das Maximum der Kinderlosigkeit noch nicht erreicht ist. Dazu gehört u.a. der steigende Anteil der Frauen mit Hochschulbildung, die eine überdurchschnittlich hohe Kinderlosigkeit aufweisen. In den neuen Ländern sowie bei westdeutschen Frauen ohne akademische Bildung wird die Kinderlosenquote in den kommenden Jahren voraussichtlich weiter zunehmen. Dadurch würde auch das Gesamtniveau der Kinderlosigkeit weiter steigen.

Sozioökonomische, biomedizinische und kulturelle Faktoren sprechen ebenfalls eher für eine Trendfortsetzung als für eine Trendwende in der Kinderlosigkeit. Durch die Einführung des Elterngelds und den Ausbau der Kinderbetreuung wurden familienpolitische Schritte unternommen, die diesem Trend entgegen wirken könn- 
ten (Bonin et al. 2013). Allerdings ist das Problem der Vereinbarkeit zwischen Beruf und Familie damit noch lange nicht gelöst (Bujard/Lück 2015a). Das immer weitere Aufschieben der Erstgeburt führt außerdem auch aus biomedizinischen Gründen dazu, dass nicht alle auf später aufgeschobenen Wünsche für mindestens ein Kind realisiert werden können (Beier et al. 2012; te Velde et al. 2012). Zugleich zeigt die jüngste Forschung zu Familienleitbildern, dass die lebenslange Kinderlosigkeit zu einem attraktiven Lebensentwurf geworden ist und "kaum noch als Defizit wahrgenommen" wird (Dorbritz/Diabaté 2015: 131).

\section{$5 \quad$ Schussfolgerungen und Diskussion}

Der Vergleich der wichtigsten Indikatoren hat gezeigt, dass das demografische Bild der Geburtenentwicklung nach dem Zensus 2011 im Wesentlichen erhalten geblieben ist. Die bisherigen Fertilitätstrends bieten deshalb eine verlässliche Ausgangsbasis für die Überlegungen zur weiteren Entwicklung der Fertilität. Diese Überlegungen sind nicht nur forschungsrelevant, die Höhe der künftigen jährlichen Geburtenziffer hat auch eine ganz reale Auswirkung auf die Geburtenzahl und damit auf die Bevölkerungsentwicklung. Bei einer zusammengefassten Geburtenziffer von etwa 1,4 Kindern je Frau ist ein deutlicher Rückgang der Geborenenzahl in naher Zukunft allein aufgrund der aktuellen Altersstruktur der weiblichen Bevölkerung vorgezeichnet (Statistisches Bundesamt 2013: 11-14). Bereits ab dem Jahr 2017 werden die immer kleiner werdenden Jahrgänge 1991 und jünger in das Alter mit höchster Fertilität von Mitte 20 bis Mitte 30 kommen. Durch die Nettozuwanderung würde die rückläufige Zahl der potenziellen Mütter voraussichtlich nur teilweise kompensiert werden können. ${ }^{4}$ Ein relativ stabiles Geburtenniveau könnte - ausgehend von den Ergebnissen der 13. koordinierten Bevölkerungsvorausberechnung - nur dann erreicht werden, wenn zusätzlich zu einer höheren Nettozuwanderung die jährliche zusammengefasste Geburtenziffer mittelfristig auf mindestens 1,6 Kinder je Frau steigen würde (Statistisches Bundesamt 2015a: Variante 6 „Relativ junge Bevölkerung").

Die Voraussetzung dafür wäre, dass die endgültige Kinderzahl je Frau auf mindestens 1,6 steigen und sich anschließend stabilisieren würde. Die bisherigen Projektionen der Kohortenfertilität zeigten eine voraussichtliche Stabilisierung der endgültigen Kinderzahl bei den Kohorten der späten 1970er Jahre auf dem Niveau leicht unterhalb 1,6 Kindern je Frau (Myrskylä et al. 2013; Statistisches Bundesamt 2015a). Um einschätzen zu können, wie wahrscheinlich ein weiterer Anstieg der Ko-

4 Die Differenz zwischen der Anzahl der 30-Jährigen und der 10-Jährigen betrug im Jahr 2013 gut 150.000. Bei einem positiven Wanderungssaldo und einer geringen Sterblichkeit wird die Zahl der heute 10-jährigen Mädchen in den nächsten 20 Jahren zunehmen. Allerdings würde sie auch bei der hohen Wanderungsannahme der 13. koordinierten Bevölkerungsvorausberechnung um maximal 60.000 steigen. Damit wäre die Zahl der 30 -jährigen potenziellen Mütter im Jahr 2033 immer noch um 90.000 kleiner als im Jahr 2013 (Statistisches Bundesamt. 13. koordinierte Bevölkerungsvorausberechnung). 
hortenfertilität wäre, untersuchten wir die Trends im Geburtenverhalten der Frauenkohorten. Im Fokus standen dabei die Auswirkungen der immer späteren Familiengründung auf die Kohortenfertilität. „In the latter part of the $20^{\text {th }}$ century and early in the $21^{\text {st }}$ century, the most prominent demographic mechanism determining fertility trends has been the extent to which childbearing postponement has been counterbalanced by birth recuperation." (Frejka/Sobotka 2008: 30). Die Erfahrungen von Frankreich oder Schweden haben beispielsweise gezeigt, dass ein steigendes Erstgeburtsalter nicht zwangsläufig zu sinkender abgeschlossener Fertilität führen muss. In Deutschland verliefen dagegen diese Entwicklungen parallel und waren offensichtlich auch die Folge eines Einstellungswandelns in Bezug auf Familie ( $E h$ mer et al. 2012). Während sich das durchschnittliche Alter beim ersten Kind bei den Kohorten ab 1947 erhöhte (Pötzsch/Sommer 2009), sank die endgültige Kinderzahl je Frau von 1,80 (Jahrgang 1946) auf 1,57 (Jahrgang 1964). Das Aufschieben der Familiengründung ging dabei mit einer schnell steigenden Kinderlosigkeit (von $12 \%$ auf $20 \%$ ) und einem sinkenden Anteil der Frauen mit 3 oder mehr Kindern von $21 \%$ auf $17 \%$ einher (Bujard/Lück 2015b: Tabelle1).

Damit die endgültige Kinderzahl trotz des Aufschubs der Familiengründung auf ein höheres Alter nicht sinkt, müsste der Rückgang der Fertilität im jüngeren Alter durch das spätere Nachholen der Geburten kompensiert oder überkompensiert werden. Mit Hilfe der Analyse des Aufschubs und des Nachholens der Geburten haben wir einen Einblick in die Interaktionseffekte der altersspezifischen Fertilität der jüngeren Jahrgänge bis 1984 gewinnen können. Dabei lässt sich zum einen die voraussichtliche leichte Erholung der endgültigen Kinderzahl bei den Jahrgängen 1969 bis 1973 erklären. Diese ist einer besonderen Konstellation zu verdanken: Die Geburtenhäufigkeit dieser Jahrgänge hat sich im jüngeren Alter unter 30 Jahren stabilisiert, während sie im Alter ab 30 Jahre weiter anstieg. Zum anderen wird offenkundig, dass die Fertilität im Alter unter 30 Jahren ab der Kohorte 1974 wieder kontinuierlich sinkt, was eine erneute Verstärkung des Geburtenaufschubs bedeutet.

Dieser Fertilitätsrückgang im jüngeren Alter kann nur dann kompensiert werden, wenn sich die Nachholtrends der letzten sechzehn Jahre linear fortsetzen würden. Neben einer deutlichen Geburtenzunahme im Alter zwischen 30 und 35 Jahren, die folgerichtig und realisierbar erscheint, bedeutet dies aber auch, dass die Fertilität im Alter ab 36 um das Vielfache steigen müsste. Aus heutiger Sicht ist dies eine sehr optimistische Annahme. Es gibt zwar noch „Reserven“ bei der Fertilität im höheren gebärfähigen Alter. So ist die Geburtenhäufigkeit der ab 36-Jährigen in Deutschland insgesamt um $26 \%$ geringer als zum Beispiel in Hamburg (Statistisches Bundesamt 2015d) und um etwa $12 \%$ geringer als in Schweden, dem Land mit dem derzeit höchsten durchschnittlichen Gebäralter in Europa (Eurostat 2015: http://ec.europa. eu/eurostat/de/data/database). Darüber hinaus weisen Goldstein et al. auf verbesserte familienpolitische Rahmenbedingungen, höhere Akzeptanz der Frauenerwerbstätigkeit und gestiegene Familienorientierung der jüngeren Geburtsjahrgänge als Faktoren hin, die eine Trendwende herbeiführen könnten (Goldstein et al. 2012). Obwohl diese Faktoren offenbar eine positive Wirkung auf die Fertilitätsentwicklung im höheren gebärfähigen Alter haben, wirken sich zugleich andere Befunde auf das Ausmaß der Kompensationseffekte im Alter ab 36 Jahre einschränkend aus. 
Insbesondere ist der Übergang zum ersten Kind im letzten Drittel der gebärfähigen Phase derzeit noch keine Selbstverständlichkeit. Der optimale Zeitpunkt für die erste Schwangerschaft einer Frau ist nach Beier et al. - biologisch gesehen - nach wie vor zwischen dem 18. und 30. Lebensjahr (Beier et al. 2012: 303). Danach sinkt die Wahrscheinlichkeit der ersten Schwangerschaft allmählich. Es bleibt abzuwarten, wie sich künftig ein weiterer medizinischer Fortschritt in den altersspezifischen Werten zur ersten Geburt niederschlagen wird. Im Hinblick auf Familienzuwachs kommen Neyer et al. zur folgenden Schlussfolgerung: „In Bezug auf dritte Geburten ergibt sich ein relativ einheitliches Bild: In allen nordischen Ländern sinkt mit steigendem Alter die Neigung von Müttern mit zwei Kindern, ein drittes zu haben [...]. [...] das relative Risiko einer zweiten Geburt fällt erst für Mütter ab Mitte 30 deutlich ab. Bei diesen Müttern kann davon ausgegangen werden, dass zumindest ein Teil von ihnen aufgrund abnehmender Fruchtbarkeit kein weiteres Kind mehr bekam, obwohl sie sich noch ein Kind wünschten." (Neyer et al. 2006). Zudem zeigen die vorgelegten Ergebnisse, dass die Mütter mit mehreren Kindern eine besondere Gruppe darstellen. Für sie ist bisher charakteristisch, ihr erstes Kind bis Mitte zwanzig zu bekommen und relativ große Abstände zwischen den einzelnen Geburten zu haben. Damit der Anteil der Mütter mit drei oder mehr Kindern auch zukünftig stabil bleibt, müssten sich diese Muster im Geburtenverhalten ändern. Nur wenn mehr Frauen, die erst im Alter ab 30 Jahre Familie gegründet haben, dritte und weitere Kinder bekommen würden und/oder das Alter bei der Familiengründung nicht mehr steigen würde, wären die Voraussetzungen dafür geschaffen. Empirisch lassen sich diese Veränderungen noch nicht nachweisen.

Ein weiteres Hindernis stellt die immer noch steigende Kinderlosigkeit dar. Um die endgültige Kinderzahl je Frau von mindestens 1,6 - bei der durchschnittlichen Kinderzahl je Mutter von 2,0 - zu erreichen und aufrechtzuhalten, dürfte die Kinderlosigkeit $20 \%$ nicht übersteigen. Im Jahr 2012 betrug die Kinderlosenquote jedoch bereits $22 \%$ und ihr Maximum ist voraussichtlich noch nicht erreicht. Trotz einzelner positiver Impulse, wie z.B. eine Verfestigung der Kinderlosenquote bei Frauen mit Hochschulabschuss auf hohem Niveau, gibt es derzeit keine Hinweise auf eine Umkehr des Trends zu höherer Kinderlosigkeit.

Zusammenfassend kann festgehalten werden: Allein die mittelfristige Stabilisierung der endgültigen Kinderzahl auf dem relativ niedrigen Niveau zwischen 1,5 und 1,6 Kinder je Frau setzt voraus, dass der Anstieg der Geburtenhäufigkeit der 1970er und 1980er Kohorten im Alter ab 30 Jahre ausreichend stark sein muss, um den zunehmenden Rückgang der Fertilität im Alter unter 30 Jahren zu kompensieren. Dafür müsste sich das Geburtenverhalten der Frauen sowohl beim Übergang zum ersten Kind als auch im Hinblick auf den Familienzuwachs verändern. Die positiven Impulse in der Geburtenentwicklung der letzten Jahre sind insofern folgerichtig und zeigen, dass sich der Nachholprozess intensiviert hat. Für einen Anstieg und anschließende Stabilisierung der Kohortenfertilität auf dem Niveau von mindestens 1,6 Kindern je Frau wäre jedoch darüber hinaus erforderlich, dass sich die Geburtenhäufigkeit im Alter unter 30 Jahren stabilisiert und die Kinderlosenquote sinkt. 


\section{Danksagung}

Die Autorin bedankt sich bei Felix zur Nieden, Bettina Sommer, Martin Bujard, Norbert $F$. Schneider und bei zwei anonymen Gutachtern für wichtige Hinweise zu früheren Versionen dieses Artikels.

\section{Literatur}

Beier, Henning M. et al. 2012: Medizinische und biologische Aspekte der Fertilität. In: Stock Günter et al. (Hrsg.): Zukunft mit Kindern: Fertilität und gesellschaftliche Entwicklung in Deutschland, Österreich und der Schweiz. Frankfurt am Main: Campus: 294-310.

Bongaarts, John; Feeney, Griffith 1998: On the quantum and tempo of fertility. In: Population and Development Review 24,2: 271-291.

Bongaarts, John; Sobotka, Tomas 2012: Demographic explanation for the recent rise in European fertility. In: Populaltion and Development Review 38,1: 83-120 [doi: 10.1111/j.1728-4457.2012.00473.x].

Bonin, Holger et al. 2013: Lehren für die Familienpolitik: Zentrale Resultate der Gesamtevaluation familienbezogener Leistungen [URL: ftp://ftp.zew.de/pub/zew-docs/ gutachten/Fampolit_Leistungen_HBO.pdf, 14.01.2016].

Bujard, Martin; Lück, Detlev 2015a: Kinderlosigkeit und Kinderreichtum: Zwei Phänomene und ihre unterschiedlichen theoretischen Erklärungen. In: BiB Working Paper 2015,1. Wiesbaden: Bundesinstitut für Bevölkerungsforschung [URN: urn:nbn:de:bibwp-2015-011].

Bujard, Martin; Lück, Detlev 2015b: Kinderlosigkeit und Kinderreichtum: Gründe und Daten für eine paritätsspezifische Fertilitätsforschung. In: Zeitschrift für Familienforschung 3,27: 255-269

Dorbritz, Jürgen; Ruckdeschel, Kerstin 2007: Kinderlosigkeit in Deutschland - Ein europäisches Sonderweg? Daten, Trends und Gründe. In: Kreyenfeld, Michaela; Konietzka, Dirk (Hrsg.): Ein Leben ohne Kinder. Wiesbaden: VS Verlag für Sozialwissenschaften: 45-82.

Dorbritz, Jürgen; Diabaté, Sabine 2015: Leitbild und Kinderlosigkeit: Kulturelle Vorstellungen zum Leben ohne Kinder. In: Schneider, Norbert F.; Diabaté, Sabine; Ruckdeschel, Kerstin (Hrsg.): Familienleitbilder in Deutschland. Verlag Babara Budrich: 113132.

Ehmer, Josef; Ehrhardt, Jens; Kohli, Martin 2012: Fertilität in historischer Perspektive. In: Stock, Günter et al. (Hrsg.): Zukunft mit Kindern. Frankfurt/New York: Campus Verlag: 41-52.

Eurostat 2015: [URL: http://ec.europa.eu/eurostat/de/data/database, 14.01.2016].

Frejka, Tomas; Calot, Gérard 2001: Cohort Reproductive Patterns in Low-Fertlity Countries. In: Population and Development Review 27,1 [doi: 10.1111/j.17284457.2001.00103.x].

Frejka, Tomas; Sobotka, Tomáš 2008: Overview Chapter: Fertility in Europe: Diverse, delayed and below replacement. In: Demographic Research 19,3 [doi: 10.4054/DemRes.2008.19.3].

Frejka, Tomas 2012: Die Auswirkungen des aktuellen Aufschubs und Nachholens von Geburten auf die Ausprägung der Periodenfertilitätstrends. In: Comparative Population Studies 36,4 [doi: 10.4232/10.CpoS-25011-20de]. 
Goldstein, Joshua R.; Sobotka, Tomáš; Jasilioniene, Aiva 2009: The end of 'lowest-low' fertility? In: Population and Development Review 35,4: 663-699 [doi: 10.1111/j.17284457.2009.00304.x].

Goldstein, Joshua R.; Kreyenfeld, Michaela 2011: Has East Germany overtaken West Germany? Recent trends in order-specific fertility. In: Population and Development Review 37,3: 453-472 [doi: 10.1111/j.1728-4457.2011.00430.x].

Goldstein, Joshua R. et al. 2011: Fertility Forecasting in the German-speaking World: Recent Experience and Opportunities for Improvement. In: Comparative Population Studies 36,2-3: 661-692 [doi: 10.4232/10.CPoS-2011-09en].

Goldstein, Joshua R.; Kreyenfeld, Michaela; Rößger, Felix 2012: Gibt es eine Trendumkehr in der Kinderzahl nach Geburtsjahrgängen in Deutschland? Working Paper: Berliner Demografie Forum, Ausgabe 4, Januar 2012.

Kreyenfeld, Michaela et al. 2010: Order-Specific Fertility Rates for Germany: Estimates from Perinatal Statistics for the Period 2001-2008. In: Comparative Population Studies 35,2: 207-224 [doi: 10.4232/10.CPoS-2010-06en].

Kreyenfeld, Michaela et al. 2012: Fertilitätsdaten für Deutschland, Österreich und die Schweiz: Wo liegen die Möglichkeiten? Was sind die Begrenzungen? In: Comparative Population Studies 36,2-3: 381-416 [doi: 10.4232/10.CPoS-2011-06de].

Konietzka, Dirk; Kreyenfeld, Michaela 2013: Kinderlosigkeit in Deutschland. Theoretische Probleme und empirische Ergebnisse. In: Konietzka, Dirk; Kreyenfeld, Michaela (Hrsg.): Ein Leben ohne Kinder: Ausmaß, Strukturen und Ursachen der Kinderlosigkeit. 2. Auflage. Wiesbaden: VS Verlag für Sozialwissenschaften.

Kohler, Hans-Peter; Ortega, José A. 2002: Tempo-adjusted period parity progression measures, fertility postponement and completed cohort fertility. In: Demographic Research 6,6: 91-144 [doi: 10.4054/DemRes.2002.6.6].

Luy, Marc 2010: Tempo-Effekte und ihre Bedeutung für die demografische Analyse. In: Comparative Population Studies 35,3: 447-482 [doi: 10.4232/10.CPoS-2010-11de].

Luy, Marc; Pötzsch, Olga 2010: Estimates of the Tempo-adjusted Total Fertility Rate in Western and Eastern Germany, 1955-2008. In: Comparative Population Studies 35,3: 569-604 [doi: 10.4232/10.CPoS-2010-14en].

Luy, Marc 2016: Demografische Kennziffern und Methoden. In: Niephaus, Yasmin; Kreyenfeld, Michaela; Sackmann, Reinhold (Hrsg.): Handbuch Bevölkerungssoziologie. Springer VS Verlag, Wiesbaden 2016.

Myrskylä, Mikko; Goldstein, Joshua R.; Cheng, Alice Yen-Hsin 2013: New Cohort Fertility Forecast for the Developed World: Rises, Falls, and Reversals. In: Population and Development Review 39,1: 31-56 [doi: 10.1111/j.1728-4457.2013.00572.x].

Neyer, Gerda R. et al. 2006: Fertilität, Familiengründung, Familienerweiterung in den nordischen Ländern. In: MPIDR Working Paper WP 2006-022 [http://www.demogr. mpg.de/papers/working/wp-2006-022.pdf, 15.01.2016].

Pötzsch, Olga; Sommer, Bettina 2009: Generatives Verhalten der Frauenkohorten im langfristigen Vergleich. In: Wirtschaft und Statistik 5/2009. Statistisches Bundesamt: Wiesbaden.

Pötzsch, Olga 2010a: Annahmen zur Geburtenentwicklung in der 12. koordinierten Bevölkerungsvorausberechnung. In: Wirtschaft und Statistik 1/2010. Statistisches Bundesamt.

Pötzsch, Olga 2010b: Kohortenfertilität: Ein Vergleich der Ergebnisse der amtlichen Geburtenstatistik und der Mikrozensuserhebung 2008. In: Comparative Population Studies 35,1: 165-184 [doi: 10.4232/10.CPoS-2010-05de]. 
Pötzsch, Olga 2013: Wie wirkt sich der Geburtenaufschub auf die Kohortenfertilität in West und Ost aus? In: Wirtschaft und Statistik 2/2013. Statistisches Bundesamt.

Prioux, France; Mazuy, Magali; Barbieri, Magali 2010: Recent demographic developments in France: fewer adults live with a partner. In: Population-E 65,3: 363-414 [doi: 10.1353/pop.2010.0016].

Ryder, Norman 1980: Components of temporal variations in American fertility. In: Hiorns, Robert W. (Hrsg.): Demographic Patterns in Developed Societies. London: Taylor and Francis: 15-54.

Sobotka, Tomáš 2003: Tempo-Quantum and Period-Cohort Interplay in Fertility Changes in Europe: Evidence from the Czech Republic, Italy, the Netherlands and Sweden. In: Demographic Research 8,6: 151-214 [doi: 10.4054/DemRes.2003.8.6].

Sobotka, Tomáš 2011: Fertility in Austria, Germany and Switzerland: Is there a Common Pattern? In: Comparative Population Studies 36,2-3 [doi: 10.4232/10.CPoS-2011-12en].

Sobotka, Tomáš et al. 2011: Postponement and Recuperation in Cohort Fertility: Austria, Germany and Switzerland in a European Context. In: Comparative Population Studies 36,2-3 [doi: 10.4232/10.CPoS-2011-10en].

Statistisches Bundesamt 2009: Imputation von Werten bei fehlenden Angaben zur Mutterschaft und zur Zahl der geborenen Kinder im Mikrozensus 2008 [https://www. destatis.de/DE/ZahlenFakten/GesellschaftStaat/Bevoelkerung/Geburten/Methoden/ Downloads/Imputationsverfahren.html, 14.01.2016].

Statistisches Bundesamt (Hrsg.) 2012: Geburten in Deutschland, Ausgabe 2012. Wiesbaden.

Statistisches Bundesamt (Hrsg.) 2013: Geburtentrends und Familiensituation in Deutschland 2012. Wiesbaden.

Statistisches Bundesamt 2014: Pressemitteilung 434/14 vom 8.12.2014 „682 000 Kinder kamen im Jahr 2013 zur Welt".

Statistisches Bundesamt (Hrsg.) 2015a: Bevölkerung Deutschlands bis 2060. Wiesbaden.

Statistisches Bundesamt 2015b: Geburtenraten und Tempoeffekt: [https://www.destatis.de/DE/ZahlenFakten/GesellschaftStaat/Bevoelkerung/Geburten/GeburtenratenTempoeffekt.html, 14.01.2016].

Statistisches Bundesamt 2015c: Daten zu Geburten, Familien und Kinderlosigkeit - Ergebnisse des Mikrozensus 2012 - Tabellen mit neuer Hochrechnung anhand der Bevölkerungsfortschreibung auf Basis des Zensus 2011 - Ausgabe 2015.

Statistisches Bundesamt 2015d: Tabellen „Altersspezifische Geburtenziffern 2014_Geburtsjahr_Länder".

Stock Günter et al. (Hrsg.) 2012: Zukunft mit Kindern: Fertilität und gesellschaftliche Entwicklung in Deutschland, Österreich und der Schweiz. Frankfurt 2012.

te Velde, Egbert et al. 2012: The effect of postponement of first motherhood on permanent involuntary childlessness and total fertility rate in six European countries since the 1970s. In: Human Reproduction 27, 4: 1179-1183 [doi: 10.1093/humrep/der455].

Wood, Jonas; Neels, Karel; Kil, Tine 2014: The educational gradient of childlessness and cohort parity progression in 14 low fertility countries. In: Demographic research 31,46: 1365-1416 [doi: 10.4054/DemRes.2014.31.46]. 
Eine Übersetzung dieses begutachteten und von der Autorin autorisierten deutschen Originaltextes durch das Bundesinstitut für Bevölkerungsforschung ist unter dem Titel "Fertility in Germany before and after the 2011 Census: Still no Trend Reversal in Sight", DOI 10.12765/CPoS-2016-02en bzw. URN urn:nbn:de:bib-cpos-2016-02en2, auf http://www.comparativepopulationstudies.de verfügbar.

Eingegangen am: 15.10.2015

Angenommen am: 29.01.2016

Olga Pötzsch ( $\bowtie)$. Statistisches Bundesamt. Wiesbaden, Deutschland.

E-Mail: olga.poetzsch@destatis.de

URL: http://www.destatis.de 


\section{Comparative Population Studies}

wWW.comparativepopulationstudies.de

ISSN: 1869-8980 (Print) - 1869-8999 (Internet)

\section{Published by}

Prof. Dr. Norbert F. Schneider

Federal Institute for Population Research D-65180 Wiesbaden / Germany

\section{(cc) BY-SA}

2017

\section{Managing Editor}

Frank Swiaczny

\section{Assistant Managing Editor}

Katrin Schiefer

\section{Copy Editor}

(Selected Articles in German)

Dr. Evelyn Grünheid

\section{Layout}

Beatriz Feiler-Fuchs

E-mail: cpos@bib.bund.de

\section{Scientific Advisory Board}

Paul Gans (Mannheim)

Karsten Hank (Cologne)

Johannes Huinink (Bremen)

Michaela Kreyenfeld (Rostock)

Marc Luy (Vienna)

Notburga Ott (Bochum)

Peter Preisendörfer (Mainz)

Nikola Sander (Groningen)

Zsolt Spéder (Budapest)

\section{Board of Reviewers}

Martin Abraham (Erlangen)

Laura Bernardi (Lausanne)

Hansjörg Bucher (Bonn)

Claudia Diehl (Konstanz)

Andreas Diekmann (Zurich)

Gabriele Doblhammer-Reiter (Rostock)

Jürgen Dorbritz (Wiesbaden)

Anette Eva Fasang (Berlin)

E.-Jürgen Flöthmann (Bielefeld)

Alexia Fürnkranz-Prskawetz (Vienna)

Beat Fux (Salzburg)

Joshua Goldstein (Berkeley)

Sonja Haug (Regensburg)

Hill Kulu (Liverpool)

Aart C. Liefbroer (The Hague)

Kurt Lüscher (Konstanz)

Emma Lundholm (Umeå)

Nadja Milewski (Rostock)

Dimiter Philipov (Vienna)

Roland Rau (Rostock)

Tomáš Sobotka (Vienna)

Jeroen Spijker (Barcelona)

Olivier Thévenon (Paris)

Helga de Valk (Brussels)

Heike Trappe (Rostock)

Michael Wagner (Cologne) 